hep-th/0203124

CTP-MIT-3251

DAMTP-2002-33

\title{
RG flows from Spin(7), CY 4-fold and HK manifolds to AdS, Penrose limits and pp waves
}

\author{
Umut Gürsoy $^{1, a}$, Carlos Núñez ${ }^{2}$, Martin Schvellinger ${ }^{1, b}$ \\ ${ }^{1}$ Center for Theoretical Physics, \\ Laboratory for Nuclear Science and Department of Physics, \\ Massachusetts Institute of Technology, \\ Cambridge, Massachusetts 02139, USA \\ ${ }^{a}$ E-mail: umut@mit.edu \\ ${ }^{b}$ E-mail: martin@lns.mit.edu \\ ${ }^{2}$ Department of Applied Mathematics and Theoretical Physics, \\ Centre for Mathematical Sciences, University of Cambridge, \\ Wilberforce Road, Cambridge CB3 0WA, U.K. \\ E-mail: C.Nunez@damtp.cam.ac.uk
}

\begin{abstract}
We obtain explicit realizations of holographic renormalization group (RG) flows from Mtheory, from $E^{2,1} \times \operatorname{Spin}(7)$ at UV to $A d S_{4} \times \tilde{S}^{7}$ (squashed $S^{7}$ ) at IR, from $E^{2,1} \times C Y 4$ at UV to $A d S_{4} \times Q^{1,1,1}$ at IR, and from $E^{2,1} \times H K$ (hyperKahler) at UV to $A d S_{4} \times N^{0,1,0}$ at IR. The dual type IIA string theory configurations correspond to D2-D6 brane systems where D6-branes wrap supersymmetric four-cycles. We also study the Penrose limits and obtain the pp-wave backgrounds for the above configurations. Besides, we study some examples of non-supersymmetric and supersymmetric flows in five-dimensional gauge theories.
\end{abstract}




\section{Introduction}

Since M-theory compactifications on manifolds of special holonomy preserve a fraction of the original supercharges in flat eleven-dimensional spacetime, it has become a fruitful arena to explore the dynamical aspects of minimally supersymmetric gauge theories. Indeed, study of special holonomy manifolds developing an isolated classical singularity has recently shed light on several important questions regarding restoration of global symmetries, phase transitions between different classical spacetimes 1, 2, 3, 4, 5, 6, 7, 8, 9, 10, 11, 12, 13, 14, 15, 16, 17, $18,19,20,21,22,23,24,25,26,27,28$, relations between anomalies in M-theory, string theory and gauge theories [3], among other relevant aspects (see for instance [6] to [26]). The dynamics of $\mathcal{N}=1$ SYM theory in four and three dimensions has been exhaustively investigated by Atiyah and Witten [2] and Gukov and Sparks [3], respectively.

Particularly, it is possible to study certain properties of these backgrounds through their dual D6 brane configurations in type IIA string theory. Any configuration of type IIA string theory with no bosonic content except than the metric, Ramond-Ramond one-form and dilaton lifts to an eleven-dimensional supergravity configuration without flux. This is a pure gravitational configuration. For instance, let us consider a collection of $N_{6}$ parallel D6-branes in type IIA string theory [29]. In eleven dimensions the metric is described by the product of a seven-dimensional Minkowski spacetime and an Euclidean multi-centered Taub-NUT space [30]. Moreover, a configuration of D6-branes in flat space can be represented in M-theory by a four-dimensional manifold with $S U(2)$ holonomy [7]. Furthermore, one can consider D6branes wrapping supersymmetric cycles in spaces with special holonomy and, as described in [7], there are two different possibilities that can be exemplified as follows. One can have D6-branes wrapping a supersymmetric four-cycle, $S^{4}$, in a $G_{2}$ holonomy manifold. Thus, D6-branes completely fill the space transverse to type IIA string theory compactification manifold, and therefore the field theory is on the transverse Minkowski three-dimensional spacetime, while the local M-theory description involves a $\operatorname{Spin}(7)$ holonomy manifold. As another example, one can consider D6-branes wrapping a different four-cycle, $S^{2} \times S^{2}$, in a CY3 fold. Then, the three-dimensional field theory is codimension one in the transverse Minkowski space to the type IIA compactification manifold. In this case, the local M-theory description is given by a CY4 fold. The corresponding pure eleven-dimensional geometric configurations were obtained long time ago in [31] and [32]. Recently, a supergravity solution was obtained when D6-branes are wrapped on $S^{4}$ in seven-dimensional manifolds of $G_{2}$ holonomy [33]. This solution preserves two supercharges and thus it represents a supergravity dual of a three-dimensional $\mathcal{N}=1 \mathrm{SYM}$ theory. Lifted to eleven dimensions this solution describes M-theory on the background of a Spin(7) holonomy manifold. A detailed analysis of the dual field theory has been done in [3]. In addition, supergravity duals of D6-branes wrapping Kahler four-cycles inside a CY3 fold have been obtained in [13]. In this case the purely gravitational M-theory description corresponds to a CY4 fold. 
A natural step forward in these investigations is to explore the role of the background four-form field strength in compactifications of M-theory on manifolds of special holonomy.

Existence of $F_{4}$ field strength will deform the geometry into a different background. In this paper we will study the situation when $F_{4}$ flux is taken on the three-dimensional Minkowski space-time plus the radial coordinate. Some questions that can be addressed are the geometry induced by this $F_{4}$ flux, the dynamical mechanism to turn on $F_{4}$ field strength and the relations among the topological cycles in M-theory, type IIA string theory and field theory in such backgrounds. The natural frame to ask these questions is eleven-dimensional supergravity . Since the corresponding gauge theory on D6-branes is a seven-dimensional one, it is actually more natural to find supergravity solutions in a simpler eight-dimensional gauged supergravity [35]. Therefore, we will find the dynamical behavior of $F_{4}$ (in the "flat directions") by solving eight-dimensional supersymmetric configurations. Then, we will perform the uplifting to eleven dimensions and study holographic RG flows in three situations. One from $E^{2,1} \times \operatorname{Spin}(7)$ at UV to $A d S_{4} \times \tilde{S}^{7}$ (squashed $S^{7}$ ) at IR, which corresponds to the first case in the classification of [7]. A second case will correspond to a flow from $E^{2,1} \times C Y 4$ fold at UV towards $A d S_{4} \times Q^{1,1,1}$ in the IR limit. Finally, we will consider the case of $E^{2,1} \times H K$ at UV towards $A d S_{4} \times N^{0,1,0}$ in the IR limit. In the IR limit, they represent duals of $\mathcal{N}=1,2$ and 3 super Yang Mills theories in three dimensions, respectively. The system under study consists of localized D2-branes inside D6-branes. We will see that, as the theory flows to the IR limit, $F_{4}$ through the "flat directions" dynamically increases. However, the number of localized D2-branes inside D6-branes remains constant, hence leading to a D2-D6 brane system. We leave the issue of exploring dynamics of the four-form field strength which lives on the four-cycle coordinates for a future investigation. An important study regarding this last $F_{4}$ configuration has been addressed in [36], although without discussing the corresponding supergravity duals.

Very recently, Berenstein, Maldacena and Nastase have proposed a compelling idea explaining how the string spectrum in flat space and pp-waves arise from the large $N$ limit of $U(N) \mathcal{N}=4$ super Yang Mills theory in four dimensions at fixed $g_{Y M}$ [37. This idea has been applied to some different backgrounds [38, 39, 40, 41, 42, 43, 44, 45, 46, 47, 48, 49, 50, 51. For all of the IR backgrounds mentioned above we will study the corresponding Penrose limit and obtain their pp-wave background. Interestingly, in each case we find the enhancement of supersymmetry from $\mathcal{N}=1,2$ and 3 to $\mathcal{N}=8$ in the dual three-dimensional SYM theory in the Penrose limit. Our examples support a similar enhancement phenomenon already found for $\mathcal{N}=1$ to $\mathcal{N}=4$ super Yang Mills theory in four dimensions [41, 42.

The paper is organized as follows. In the next section we will describe the general idea and motivations. In section 3 we describe some generalities of the D2-D6 brane system in

\footnotetext{
${ }^{1}$ Also, considering the standard issues in the duality between type IIA string theory and elevendimensional supergravity, the correspondence between certain degrees of freedom in type IIA string theory and M-theory gives evidence to suspect that this duality goes beyond supergravity approximation [34].
} 
the flat case. In section 4 we obtain an RG flow from Spin(7) holonomy manifolds at UV to AdS spaces at IR and, also discuss the field theory duals. Then, in section 5 we will consider flows from $\mathrm{CY} 4$ folds to AdS spaces and a case preserving $\mathcal{N}=3$ supersymmetries in three dimensions. Section 6 is devoted to an analysis of the Penrose limits in the IR region of the supergravity solutions mentioned above. In section 7 we will study some examples of non-supersymmetric and supersymmetric flows in five-dimensional gauge theory. These flows will also be of interest since their uplifting to massive type IIA is known [52]. Appendix A introduces eight-dimensional gauged supergravity and discusses its relevant aspects related to our present interest. In Appendix B we present more general super-kink solutions of BPS equations which include above as special cases and consider their dual RG flow interpretation. In Appendix $\mathrm{C}$ we show some numerical solutions. Finally, in Appendix D we introduce some notation for the squashed seven-sphere.

\section{General idea}

As mentioned in the introduction, we will find supergravity solutions describing the RG flow from a special holonomy manifold (Spin(7) or CY4 folds) to manifolds of the form $A d S_{4} \times \tilde{M}_{7}$. We can understand these flows by realizing the fact that, since three-dimensional gauge theories have a dimensionful coupling constant, they flow to interacting IR fixed points [53, 54, 55]. These flows are interesting by their own, since they realize new examples of $\mathrm{AdS} / \mathrm{CFT}$ correspondence and some generalizations of it.

The way in which we will find our solutions is the following: we will start from the eight-dimensional $S U(2)$ gauged supergravity [35], that was proven to descend from elevendimensional supergravity as a reduction on $S^{3}$ (where only one of the two $S U(2)$ s is being gauged). We will find the solutions in this lower dimensional supergravity, and then lift them to eleven dimensions.

The advantage of doing the computations in this way is that, working with a delocalized D2-D6 system, in principle, one has to deal with a seven-dimensional gauge theory, hence one is naturally led to consider an eight-dimensional gravity theory. Indeed, we will see that after lifting, our solutions represent either D6 branes or a system of D2-D6 branes. Then, we will wrap D6 branes on some supersymmetric cycle, leading to a localized D2-D6 system. As it is well known, when a brane wraps a supersymmetric cycle, there is a way to preserve some amount of supersymmetry through the so-called twisting mechanism [56]. Realization of this mechanism in supergravity is basically the equality (here we suppress gamma matrices) of the spin connection of the manifold and the gauge field of the gauged supergravity under study, i.e. $\omega_{\mu}=A_{\mu}$, such that this combination is canceled in the covariant derivative, and thus allowing one to define a covariantly constant Killing spinor everywhere on the brane. Many interesting realizations of this mechanism have been previously worked out (see [57] to [72]). 
We will construct solutions where D6-branes are wrapping a four-cycle $\left(S^{4}\right)$ inside a $G_{2}$ holonomy manifold, and a second set of solutions where D6-branes wrap a four-cycle $\left(S^{2} \times S^{2}\right)$ inside a CY3 fold. Also, we consider an example preserving $\mathcal{N}=3$ supersymmetries in three dimensions. These examples realize M-theory configurations preserving $\mathcal{N}=1, \mathcal{N}=2$ and $\mathcal{N}=3$ supersymmetries in three dimensions, i.e. two, four and six supercharges respectively.

\section{The system under study}

As mentioned above, we will firstly study a delocalized D2-D6 brane system. In order to see this explicitly from a metric description, let us construct solutions in eight-dimensional supergravity where the field content will be a dilaton $\phi(r)$, a four-form field $G_{4}$ and a metric of the form,

$$
\begin{gathered}
d s_{8}^{2}=e^{2 f} d x_{1,2}^{2}+d r^{2}+e^{2 h} d \vec{y}_{4}^{2}, \\
G_{x_{1} x_{2} x_{3} r}=\Lambda e^{-4 h-2 \phi},
\end{gathered}
$$

where $d x_{1,2}^{2}$ is the flat Minkowski metric in 3 dimensions. In Eq.(21) we have written the four-form field in flat indices. In that follows we will assume the scalar functions $f, h, \phi$ (and also $\lambda$ ) to be only $r$-dependent.

Plugging this configuration into the supersymmetric variations of the fermion fields and requiring these variations to vanish, one can obtain a system of BPS equations (where prime denotes derivative with respect to $r$ )

$$
\begin{aligned}
f^{\prime} & =-\frac{1}{8} e^{-\phi}-\frac{\Lambda}{2} e^{-4 h-\phi}, \\
\phi^{\prime} & =-\frac{3}{8} e^{-\phi}+\frac{\Lambda}{2} e^{-4 h-\phi}, \\
h^{\prime} & =-\frac{1}{8} e^{-\phi}+\frac{\Lambda}{2} e^{-4 h-\phi} .
\end{aligned}
$$

Following the prescription given in ref.[35], one can easily see that after lifting the solutions of the system above, they will correspond to M-theory configurations of the form

$$
\begin{gathered}
d s_{11}^{2}=e^{2 f-2 \phi / 3} d x_{1,2}^{2}+e^{-2 \phi / 3} d r^{2}+e^{2 h-2 \phi / 3} d \vec{y}_{4}^{2}+4 e^{4 \phi / 3} d \Omega_{3}^{2}, \\
F_{x_{1} x_{2} x_{3} r}=2 \Lambda e^{-4 h-2 \phi / 3},
\end{gathered}
$$

where again we have used flat indices for the four-form field strength.

Now, we want to interpret the equations above as describing a D2-D6 brane system. Indeed, by setting $\Lambda$ equal to zero, the solution is given by the metric corresponding to D6-branes in the near horizon region (lifted to M-theory) [73]. On the other hand, if we 
consider non-vanishing $\Lambda$, we can compute a solution that shows the presence of D2-branes delocalized inside the D6-brane worldvolume. In this case the M-theory solution is

$$
\begin{gathered}
d s_{11}^{2}=\frac{\rho^{2}}{36} d x_{1,2}^{2}+\frac{4 \sqrt{\Lambda}}{9 \rho} d y_{4}^{2}+9^{3} d \rho^{2}+81 \rho^{2} d \Omega_{3}^{2}, \\
F_{x_{1} x_{2} x_{3} \rho}=\frac{1}{18 \rho} .
\end{gathered}
$$

This solution is the near horizon limit of the one obtained in [74.

\section{From Spin(7) holonomy manifolds to AdS spaces and FT duals}

\subsection{D6-branes wrapping cycles inside $G_{2}$ holonomy manifolds}

In this section we will consider D6-branes wrapping a four-sphere in a $G_{2}$ holonomy manifold2]. Furthermore, we will add D2-branes in the unwrapped directions. After the twisting is performed we obtain a $2+1$-dimensional gauge theory with 2 supercharges, i.e. $S U(N)$ $\mathcal{N}=1$ SYM theory in three dimensions.

Our solution will describe a flow of this theory from a Spin(7) holonomy manifold to an AdS manifold, thus realizing the flow towards the IR fixed point that this kind of theories has, but in a gravitational set-up. We found a second class of solutions which we included in Appendix B. However, these have singularities which makes investigation of the field theory duals more difficult.

As we have already mentioned, when D6-branes wrap a cycle, in order to preserve some amount of supersymmetries we have to do a twisting procedure in the seven-dimensional gauge theory. In the present case we perform the twisting with an $S U(2)$ gauge connection, and choosing the four-cycle to be a four-sphere, we will have an $S U(2)$ instanton on $S^{4}$.

As it is well known, when we wrap D6-branes on a curved cycle there will be an induced D2-brane charge, that can be understood as coming from the WZ coupling in the Born-Infeld action [76], of the form

$$
\int_{E^{2,1} \times S^{4}} C_{3} \wedge\left(F_{2} \wedge F_{2}+R_{2} \wedge R_{2}\right)
$$

The second term leads to an induced D2-brane charge similar to the one that we propose in our configuration and it can be understood as an effective cosmological constant. When we turn on an $F_{4}$ flux in the four-cycle, the first term induces a Chern-Simons term in the

\footnotetext{
${ }^{2}$ We consider non-compact manifolds with special holonomy. For the construction of compact manifolds with special holonomy see 75 .
} 
$2+1$ field theory. We postpone the study of this last type of interesting configurations for the future.

Let us consider a metric description for this field configuration. In eight-dimensional supergravity we can use the following metric ansatz

$$
d s_{8}^{2}=e^{2 f} d x_{1,2}^{2}+d r^{2}+e^{2 h} d \Omega_{4}^{2},
$$

while in flat indices the four-form field strength is defined as in Eq.(2)). Then, the BPS equations become

$$
\begin{aligned}
f^{\prime} & =\frac{1}{8} e^{-\phi}-e^{\phi-2 h}+\frac{\Lambda}{2} e^{-4 h-\phi}, \\
\phi^{\prime} & =\frac{3}{8} e^{-\phi}-3 e^{\phi-2 h}-\frac{\Lambda}{2} e^{-4 h-\phi}, \\
h^{\prime} & =\frac{1}{8} e^{-\phi}+2 e^{\phi-2 h}-\frac{\Lambda}{2} e^{-4 h-\phi} .
\end{aligned}
$$

Since when $\Lambda=0$ the system above reduces to the one studied in [33], we will have the same solution, namely a cone over weak $G_{2}$. This solution is singular at IR and this singularity can be resolved by considering more elaborate solutions, in our case, this is basically attained by including an integration constant, such that the complete solution reads

$$
d s^{2}=d x_{1,2}^{2}+\frac{9}{20} \rho^{2} d \Omega_{4}^{2}+\frac{9 \rho^{2}}{100}\left(1-\left(\frac{a}{\rho}\right)^{10 / 3}\right)(\omega-A)^{2}+\frac{d \rho^{2}}{\left(1-\left(\frac{a}{\rho}\right)^{10 / 3}\right)},
$$

which is the metric of a $\operatorname{Spin}(7)$ holonomy manifold having the topology of an $\mathbf{R}^{4}$ bundle over $S^{4}$.

After the appropriate modding out by $Z_{N}$, this metric describes the M-theory version of the gravitational side of the geometrical transition between $N_{6}$ D6-branes wrapping a four-cycle inside a $G_{2}$ manifold and a situation without branes but flux over a two-cycle. Solutions similar to these ones, but with a stable $U(1)$ at long distances, were recently studied in [8, 9, 12].

We can try to achieve a different type of resolution, by turning on another degree of freedom in M-theory, namely the $F_{4}$ field, that is $\Lambda$ being non-zero in the system (12)(14). Obviously, this will take us out from the "pure metric" configurations, but the type of resolution is well-behaved and it describes a phenomenon that seems to be a common characteristic in three-dimensional gauge theories. In the following, we are going to introduce different solutions of the above BPS equations, and discuss them in terms of their ability in describing physically meaningful holographic RG flows. 
A solution driving the flow from $E^{2,1} \times \operatorname{Spin}(7)$ to $A d S_{4} \times \tilde{S}^{7}$

Through the following change of variables

$$
d r=e^{\phi} d \tau
$$

one can find a solution that reads

$$
\begin{aligned}
h(\tau) & =\frac{1}{4} \log \left(\frac{20 \Lambda}{9}+\frac{A}{9} e^{9 \tau / 10}\right), \quad \phi(\tau)=-1 / 2 \log (20)+h(\tau), \\
f(\tau) & =\frac{3 \tau}{10}-\frac{1}{4} \log \left(20 \Lambda+A e^{9 \tau / 10}\right),
\end{aligned}
$$

where $A$ is an integration constant. The corresponding eleven-dimensional metric is

$$
\begin{aligned}
d s_{11}^{2}= & \frac{60^{1 / 3} e^{3 \tau / 5}}{\left(A e^{9 \tau / 10}+20 \Lambda\right)^{2 / 3}} d x_{1,2}^{2}+\left(\frac{2 \sqrt{5}}{3}\right)^{2 / 3}\left(A e^{9 \tau / 10}+20 \Lambda\right)^{1 / 3} d \Omega_{4}^{2} \\
& +\left(\frac{2}{15}\right)^{2 / 3}\left(A e^{9 \tau / 10}+20 \Lambda\right)^{1 / 3}\left(\omega^{i}-A^{i}\right)^{2}+\frac{1}{(2 \sqrt{15})^{4 / 3}}\left(A e^{9 \tau / 10}+20 \Lambda\right)^{1 / 3} d \tau^{2}
\end{aligned}
$$

while in flat indices the four-form field strength is

$$
F_{x_{1} x_{2} x_{3} \tau}=\frac{18(2 \sqrt{15})^{2 / 3} \Lambda}{\left(A e^{9 \tau / 10}+20 \Lambda\right)^{7 / 6}} .
$$

In order to obtain the M-theory configuration we have used the Salam-Sezgin's prescription to lift the metric and the four-form field strength to eleven dimensions. It is interesting to note that although we obtained the solution (18) from an eight-dimensional perspective, it can be recognized as the well-known M2-brane solution with fewer supersymmetries to eleven-dimensional supergravity whose existence was first proved in [77] and, its explicit form was obtained in [78]. Equivalence of solution (18) with the M2-brane solution can be seen by changing the radial variable as $r=e^{3 \tau / 20}$. This is further checked below by comparing the mass spectrum obtained from eight-dimensional BPS equations in the IR limit with the spectrum of $A d S_{4} \times \tilde{S}^{7}$ compactification of eleven-dimensional supergravity [79].

Let us now try to understand the two regimes described by this metric, by analyzing the UV and IR limits. For large values of $\tau$ we have $\lim _{\tau \rightarrow+\infty} h(\tau)=+\infty$, leading to a large radius for the four-sphere. Besides, the Ricci scalar $R$ vanishes as $\tau \rightarrow+\infty$. Changing variables again $[$ in the limit of large $\tau$ the metric (18) becomes the cone over the squashed (weak $G_{2}$ ) Einstein seven-sphere, i.e. the large distance limit of the $\operatorname{Spin}(7)$ holonomy manifold given in [33, 31, 3]

$$
d s_{11}^{2}=d x_{1,2}^{2}+d \rho^{2}+\frac{9}{20} \rho^{2} d \Omega_{4}^{2}+\frac{9}{100} \rho^{2}\left(\omega^{i}-A^{i}\right)^{2},
$$

\footnotetext{
${ }^{3}$ We use $\rho=\frac{20}{3} \frac{A^{1 / 6}}{2^{2 / 3}(15)^{1 / 3}} e^{3 \tau / 20}$.
} 
while, of course, $F_{x_{1} x_{2} x_{3} r}=0$. In figure 1 we show the behavior of $F_{x_{1} x_{2} x_{3} r}, 2 / 3 F_{x_{1} x_{2} x_{3} r}^{2}$ and the Ricci scalar $R$, as a function of $\tau$. We can see how the solid lines are exactly the same curve up to a minus sign. This graphically reflects the validity of the eleven-dimensional equations of motion.

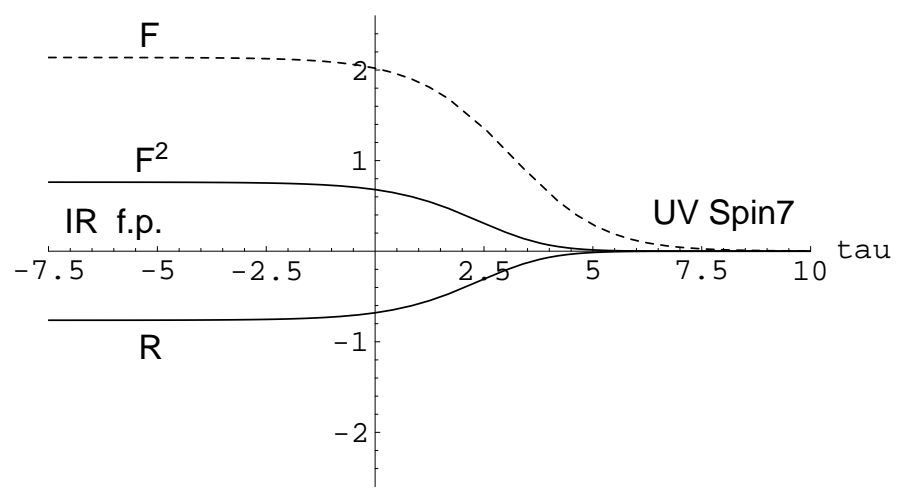

Figure 1: $F_{x_{1} x_{2} x_{3} r}, 2 / 3 F_{x_{1} x_{2} x_{3} r}^{2}$ (labeled as $F^{2}$ ) and the Ricci scalar $R$, as a function of $\tau$.

From this figure, we can also see how both the four-form field strength and the Ricci scalar go to zero at the UV limit. From the vanishing of the Ricci scalar it shows how the eleven-dimensional manifold becomes flat at UV, while in the IR limit it is negative, as one expects from AdS-like spacetimes.

We can understand some aspects of the field theory at the UV regime described by the metric (20). A very beautiful paper analyzing these kind of aspects is [3]. We can understand topological objects in the effective $2+1$-dimensional field theory as follows. From an Mtheory perspective they must correspond to M2 or M5-branes wrapping non-contractible cycles, that "intersect" the 2+1-dimensional worldvolume. Existence of suitable non-trivial cycles will signal the possibility of having a given topological defect. For example, a domain wall will be represented by an M5-brane wrapping a four-cycle, so the existence of domain walls will be determined by non-triviality of $H_{4}\left(X_{8}, Z\right)$ (where $X_{8}$ is the eight-dimensional manifold "external" to $2+1$ flat directions). Monopoles and instantons will be associated with $H_{5}\left(X_{8}, Z\right)$ and $H_{3}\left(X_{8}, Z\right)$ since they will correspond to M5-branes wrapping five-cycles and membranes wrapping three-cycles. Indeed, there is a correspondence between M-theory and type IIA string theory degrees of freedom. For instance, if one considers the multi-centered Taub-NUT metric times a seven-dimensional Minkowski spacetime in eleven dimensions, in type IIA string theory one can think of that as $n+1$ parallel D6-branes placed at each center $r_{i}$ of the Taub-NUT four-dimensional metric. In eleven dimensions the $A_{n}$ singularity can 
be resolved by $n$ homologically non-trivial cycles at $r_{i}$. Therefore, there are $n$ normalizable cohomological two-forms, $\omega^{i}$. Also there is an additional normalizable two-form $\omega^{0}$ with no topological meaning. Then, the expansion of the Ramond-Ramond three-form of type IIA string theory can be done as

$$
C_{(3)}=\sum_{i=0}^{n} \omega^{i} \wedge A_{i} .
$$

It involves an $U(1)$ seven-dimensional gauge field localized at the center of the Taub-NUT space, and it corresponds to the $U(1)$ gauge field on each D6-brane. In addition, there are $n(n+1) / 2$ holomorphic embeddings of two-cycles in the mentioned Taub-NUT metric, and an M2-brane wrapped on any of these is a BPS particle, while in type IIA string theory it becomes a string stretched between two D6-branes [34].

As explained in [3], metrics like the one in Eq.(18) are very good classical backgrounds, but they fail to give a good description of the quantum theory. Indeed, they suffer membrane anomalies. The absence of $F_{4}$ flux in the curved part $\left(S^{4}, S^{2} \times S^{2}\right.$ and a $C P^{2}$ manifold, in this paper) turns out to be the source of global membrane anomalies [80, 81]. Our metrics do not cure this problem, however they are a step towards its resolution by dynamically turning on the $F_{4}$ field. In this case this flux gives a number of localized D2-branes inside D6-branes in type IIA string theory. In eleven-dimensional supergravity this number of M2-branes (following the notation of [3] we will denote it by $N_{M 2}$ ) will have a very interesting effect on the system. These D2-branes are instantons from the viewpoint of the $\mathcal{N}=4, d=4$ twisted topological super Yang Mills theory living on the curved part of the D6-branes, hence they must encode the information of the moduli space of $N_{M 2}$ instantons.

When the number of D2/M2-branes is zero (the case when $\Lambda=0$ in our BPS system) there are no dynamical scalars in the worldvolume theory. This can be seen in two ways. First, one observes that D6-branes wrapping a four-cycle in a $G_{2}$ holonomy manifold do not leave us with flat transverse directions where the brane would in principle fluctuate. Also, no hypermultiplets are present since there are no massless modes that could be excited on the four-sphere. Secondly, following [2] one can compute the fluctuations of the metric and see that they are not square integrable in the eight-dimensional manifold, rendering the fluctuations non-dynamical which should be interpreted as a coupling constant. As we will see in the next section, this situation changes when we consider the $\mathcal{N}=2$ version of this set up. In that case one real scalar field will be dynamical and together with the vector field, it will fill the $\mathcal{N}=2$ supermultiplet.

Nevertheless, as mentioned above, in our system where the $C_{3}$ field is excited additional dynamical scalars will appear. These scalars will encode the information on the instantons in the topological field theory on $S^{4}$. Indeed, these scalars do not have a purely geometrical origin. If this were the case, they would not have dynamics and should just represent a coupling constant.

Now, we should clarify a point. In the UV limit, we do not expect the theory to be strictly 
a three-dimensional one, since likely at very high energies there will be massive modes excited on the cycle $S^{4}$, however, one can think about that as doing a fine-tuning of the constants of the problem, that is the strength $(\Lambda)$ of the $F_{4}$ field and the integration constant $A$ such that, even when we are at high energies, the theory is nearly three-dimensional. In any case the existence of the topological objects described above and other features are independent of the scale of energy at which we are observing the theory.

Our complete metric Eq.(18) describes the flow between a theory with the characteristics mentioned above and a supersymmetric conformal field theory, described by the fixed point solution we study bellow. The gravitational background is that of M2-branes on the tip of an $\operatorname{Spin}(7)$ cone. In order to see this, we will consider the situation in which the radial variable $\tau$ takes very large negative values $(\tau \rightarrow-\infty)$ and we can see in this limit how the solution reaches the fixed point, i.e. $A d S_{4} \times \tilde{S}^{7}$,

$$
d s_{11}^{2}=\left(\frac{3}{20 \Lambda^{2}}\right)^{1 / 3} e^{3 \tau / 5} d x_{1,2}^{2}+\left(\frac{\Lambda}{180}\right)^{1 / 3} d \tau^{2}+\left(\frac{20}{3}\right)^{2 / 3} \Lambda^{1 / 3} d \Omega_{4}^{2}+\left(\frac{16 \Lambda}{45}\right)^{1 / 3}\left(\omega^{i}-A^{i}\right)^{2} .
$$

As usual $\omega^{i}$ are the left-invariant one-forms defined on the $S U(2)$ group manifold, while $A^{i}$ corresponds to an $S U(2)$-instanton with only non-vanishing components on the four-sphere. Both the Ricci scalar and the four-form field strength become constant

$$
F_{x_{1} x_{2} x_{3} \tau}=\frac{9 \cdot 3^{1 / 3}}{2^{2 / 3} 5^{5 / 6} \Lambda^{1 / 6}}
$$

It is staightforward to work out the scale dimensions of the operators in the corresponding CFT at IR. This is most easily done by linearizing Eqs.(14) and (13) near the fixed point

$$
h=\frac{1}{4} \log \left(\frac{20 \Lambda}{9}\right), \quad \phi=-1 / 2 \log (20)+h .
$$

The eigenvectors of the mass matrix turn out to be, $\varphi_{1} \equiv \phi-h$ and $\varphi_{2} \equiv \phi+6 h$ whose linearized solutions read,

$$
\varphi_{1}(r) \approx A_{1} e^{-\frac{4}{3} r}, \quad \varphi_{2}(r) \approx A_{2} e^{3 r}
$$

which shows that scale dimensions of the correspoding operators in dual CFT read,

$$
\Delta_{\varphi_{1}}=6, \quad \Delta_{\varphi_{2}}=\frac{4}{3} \text { or } \frac{5}{3}
$$

The ambiguity in the second dimension is due to two possible unitary deformations of the CFT with a source or VEV [82]. The corresponding masses are $M_{1}^{2}=-\frac{80}{9} m^{2}, M_{2}^{2}=$ $+72 \mathrm{~m}^{2}$ in mass unit $\mathrm{m}^{2}$ set by the radius of $A d S_{4}$. One can easily see from the uplifting formulae in Appendix A that, $\varphi_{1}(r)$ and $\varphi_{2}(r)$ correspond to "squashing" and "overall size" deformations of $\tilde{S}^{7}$, respectively. Therefore, we see that "squashing" is a relevant whereas 
"overall size" is an irrelevant deformation in the dual CFT. In 83] the squashing operator, $\varphi_{1}$ was identified with the singlet of the isometry group $S O(5) \times S O(3)$ of $\tilde{S}^{7}$ under the decomposition of $(0,2,0,0)$ of $S O(8)$. Obviously the overall size operator, $\varphi_{2}$ should be identified with the singlet of $S O(5) \times S O(3)$ inherited from $(0,0,0,0)$ of $S O(8)$. Looking at the standard tables for mass operators, e.g. in [84], one indeed sees that it is the singlet in the KK-tower $0^{+(3)}$ and has $M_{2}^{2}=+72 \mathrm{~m}^{2}$ and energy $\Delta=6$. Note that in our solution (17) we turned off the squashing deformation, i.e. we set $h-\phi=$ const. This is a supersymmetric hence stable truncation. Since the only relevant deformation in the dual theory is turned off and we are left with an irrelevant overall size deformation, CFT appears in IR. On the other hand, in the more general solution of Appendix B where we turn on $\varphi_{1}$ along with $\varphi_{2}$, dual CFT appears in the UV limit, as expected.

It is interesting to plot the solution in terms of the variables introduced in [59], $H=s^{2} e^{-2 \phi}$ and $s=e^{2 h}$. These variables are choosen in order to study the orbits of the ODEs

$$
\frac{d H}{d s}=\left(\frac{-H s^{2}+56 s^{3}-4 \Lambda H}{H s^{2}+16 s^{3}-4 \Lambda H}\right) \frac{H}{s},
$$

obtained from Eqs.(13) and (14). Figure 2 shows the solution (17) in the variables $s$ and $H$, which is represented by a solid line.

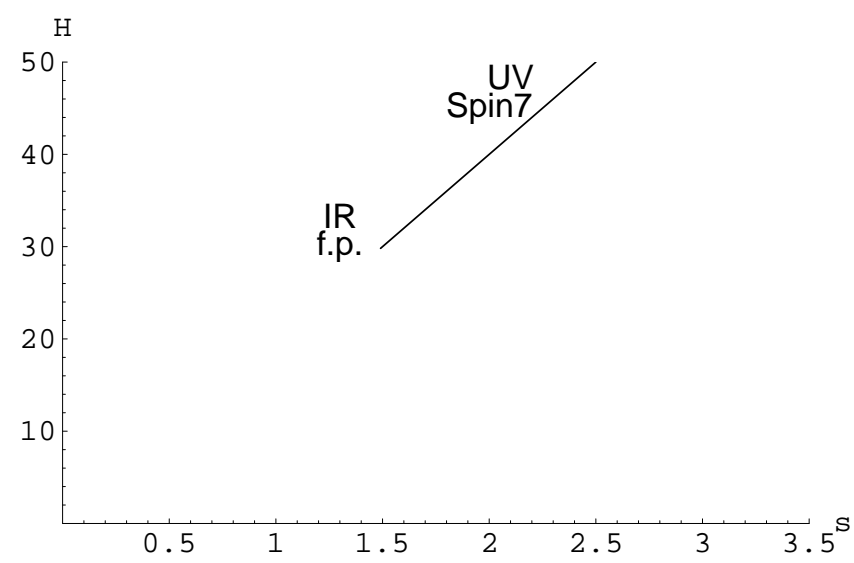

Figure 2: Solution as given by Eq.(17). The vertical axis is $H(s)$ and the horizontal one is $s$.

If one approaches to the fixed point at the end of the semi-infinite line $H=20 \mathrm{~s}$, one obtains that $f \rightarrow-\infty$ which confirms the IR character of the fixed point. Note that in the figure we fixed $\Lambda=1$ and one can easily see that as $\Lambda$ increases, the IR end-point moves upwards along $H=20 \mathrm{~s}$. In Appendix $\mathrm{C}$ we show a more detailed plot of the orbits of Eq.(27). 
The conclusion is that at UV the metric asymptotically corresponds to a three-dimensional Minkowski spacetime times an Spin(7) holonomy manifold. Then, it flows to the IR fixed point $\left(A d S_{4} \times \tilde{S}^{7}\right)$ along $H=20 s$.

One can consider more general solutions to the BPS equations (12)-(14) by turning on one more degree of freedom, namely allowing also the difference $\phi(\tau)-h(\tau)$ in Eq. (17) to be $\tau$-dependent. This solution and similar generalizations of the solutions of next section are obtained in Appendix B. They typically suffer from curvature singularities which render the field theory interpretation difficult. However, we present some cases where curvature singularities at IR are acceptable by Gubser's criterion [85].

\section{$5 \quad$ From CY4 folds to AdS spaces and FT duals}

\subsection{D6-branes wrapping cycles inside CY3 folds}

Now, we will study a system in an analogous way as in the previous section, but preserving twice the number of supersymmetries compared to the above $E^{2,1} \times \operatorname{Spin}(7)$ example. Therefore, we will consider a D2-D6 system where the D6-branes are wrapping a four-cycle of constant curvature which, in the present case, will be $S^{2} \times S^{2}$. At the UV limit this fourcycle is inside a CY3 fold, and the number of supercharges preserved by this configuration becomes 4 , thus leading to an $\mathcal{N}=2 \mathrm{SYM}$ theory in three dimensions. On the other hand, in the IR limit we will obtain an eleven-dimensional metric corresponding to M2-branes on the tip of cone based over $Q^{1,1,1}$, an Einstein-Sasakian manifold. This represents the gravity dual of a three-dimensional $\mathcal{N}=2 \mathrm{SCFT}$. In this section we want to argue that special holonomy manifold is a cone over a CY3 fold. Furthermore, we will see that it can be resolved in two possible ways, both of them require to turn on an additional degree of freedom in the gauged supergravity. First way of resolution preserves the zero curvature of the configuration and leads to special holonomy manifolds representing duals of three-dimensional $\mathcal{N}=2$ gauge theories with a mass gap, whereas the other way turns on a degree of freedom in the lower dimensional supergravity leading to a configuration of the form $A d S_{4} \times Y_{7}$ in M-theory. Here our interest concentrates on the latter type of resolutions, that as in the previous section, leads to different physical effects.

Let us start by considering the theory with D2 and D6-branes. Since we want to wrap the D6-branes in a four-cycle of the form $S^{2} \times S^{2}$, we have to choose an eight-dimensional metric of the form

$$
d s_{8}^{2}=e^{2 f} d x_{1,2}^{2}+d r^{2}+e^{2 h}\left(d \theta_{1}^{2}+\sin ^{2} \theta_{1} d \varphi_{1}^{2}+d \theta_{2}^{2}+\sin ^{2} \theta_{2} d \varphi_{2}^{2}\right),
$$

together with an Abelian gauge field $A^{(3)}=\cos \theta_{1} d \varphi_{1}+\cos \theta_{2} d \varphi_{2}$. It means that now the normal bundle of $S^{2} \times S^{2}$ is $U(1)$. Therefore, in order to define Killing spinors by means of twisting, we must break the $S U(2)$ group down to $U(1)$. This is achieved by turning on the 
field $\lambda(r)$ in the eight-dimensional gauged supergravity. Indeed, this field makes a distinction between the three directions of the $S^{3}$ "external" to the branes system, thus leading to a symmetry breaking $S U(2) \rightarrow U(1)$. For this reason, in gauged supergravity we choose a "vielbein" of the form $L_{\alpha}^{i}=\operatorname{diag}\left(e^{\lambda}, e^{\lambda}, e^{-2 \lambda}\right)$, that generates $T^{11}=T^{22}=e^{2 \lambda}, T^{33}=e^{-4 \lambda}$, as well as,

$$
\begin{gathered}
P_{\mu}^{11}=P_{\mu}^{22}=\partial_{\mu} \lambda, \quad P_{\mu}^{33}=-2 \partial_{\mu} \lambda \\
Q_{\mu}^{12}=-g A_{\mu}^{(3)}, \quad Q_{\mu}^{13}=g \cosh (3 \lambda) A_{\mu}^{(2)}, \quad Q_{\mu}^{32}=g \sinh (3 \lambda) A_{\mu}^{(1)} .
\end{gathered}
$$

As before, we have a $G_{4}$ field (in flat index notation)

$$
G_{x_{1} x_{2} x_{3} r}=\Lambda e^{-2 \phi-4 h}
$$

After plugging it into the supersymmetric variations of fermions, it produces the following BPS equations,

$$
\begin{gathered}
f^{\prime}=-\frac{1}{3} e^{\phi-2 h-2 \lambda}+\frac{1}{24} e^{-\phi}\left(e^{-4 \lambda}+2 e^{2 \lambda}\right)+\frac{\Lambda}{2} e^{-4 h-\phi} \\
\phi^{\prime}=-e^{\phi-2 h-2 \lambda}+\frac{1}{8} e^{-\phi}\left(e^{-4 \lambda}+2 e^{2 \lambda}\right)-\frac{\Lambda}{2} e^{-4 h-\phi} \\
h^{\prime}=\frac{2}{3} e^{\phi-2 h-2 \lambda}+\frac{1}{24} e^{-\phi}\left(e^{-4 \lambda}+2 e^{2 \lambda}\right)-\frac{\Lambda}{2} e^{-4 h-\phi} \\
\lambda^{\prime}=\frac{2}{3} e^{\phi-2 h-2 \lambda}-\frac{1}{12} e^{-\phi}\left(-2 e^{-4 \lambda}+2 e^{2 \lambda}\right) .
\end{gathered}
$$

When $\Lambda=0$ this system coincides with the one in ref. [13] 1 .

Indeed, in this case $(\Lambda=0)$, a simple solution can be easily found

$$
\lambda=\frac{1}{6} \log (2), \quad e^{h}=\frac{3 r}{4 \sqrt{2}}, \quad \phi=h-\frac{7}{6} \log (2), \quad 3 f=\phi
$$

that lifted to eleven dimensions, and after a suitable change of radial variable, reads as

$$
\begin{aligned}
d s_{11}^{2}= & d x_{1,2}^{2}+d \rho^{2}+ \\
& \frac{\rho^{2}}{8}\left(d \Omega_{1}^{2}+d \Omega_{2}^{2}+d \Omega_{3}^{2}+\frac{1}{2}\left(d \psi+\cos \alpha d \beta-\cos \theta_{1} d \phi_{1}-\cos \theta_{2} d \phi_{2}\right)^{2}\right) .
\end{aligned}
$$

The metric has a singularity at $\rho=0$. We are mainly interested in resolving the singularity by turning on M2-branes. However, as an aside, let us consider other ways of resolution which do not render the field theory ending in a conformal point as follows. In gauged supergravity it can be done giving the field $\lambda$ a radial dependence. Indeed, by allowing $\lambda$ to

\footnotetext{
${ }^{4}$ We thank the authors for clarifying a missprint in their original version.
} 
be variable we can obtain a more general solution that can be continued towards the IR. By defining a function $w$ and changing variables like

$$
w(\rho)=\frac{3 \rho^{4}+8 \rho^{2}+6+C \rho^{-4}}{6\left(\rho^{2}+1\right)^{2}}, \quad d r=d \rho\left(\frac{\rho^{2}}{16 w^{5 / 3}}\right)^{1 / 4}
$$

we have a solution that reads

$$
\begin{aligned}
e^{-6 \lambda} & =w(\rho), \quad e^{2 / 3 \phi}=\frac{\rho w^{1 / 6}}{4}, \\
e^{2 h} & =\frac{\left(\rho^{2}+1\right) \rho w^{1 / 6}}{16}, \quad f=\frac{1}{3} \phi .
\end{aligned}
$$

This generates an eleven-dimensional metric as

$$
\begin{aligned}
d s_{11}^{2}= & d x_{1,2}^{2}+\frac{1}{w(\rho)} d \rho^{2}+\frac{\rho^{2}}{4} d \Omega_{1}^{2} \\
& \left.+\frac{\left(\rho^{2}+1\right)}{4}\left(d \Omega_{2}^{2}+d \Omega_{3}^{2}\right)+\frac{\rho^{2} w(\rho)}{4}\left(d \psi+\cos \alpha d \beta-\cos \theta_{1} d \phi_{1}-\cos \theta_{2} d \phi_{2}\right)^{2}\right),
\end{aligned}
$$

corresponding to the metric for $\mathbf{C}^{2}$ bundle over $C P^{1} \times C P^{1}$. In the case in which the integration constant $C=0$ we recover the solution given in [13, 14], in the case of non-zero $C$ this seems to be another possible resolution.

Now, let us turn back to our main interest, resolution by dynamically turning on $F_{4}$. To this end, we will analyze a different kind of solutions of the above BPS equations, with a non-zero parameter $\Lambda$ and study their holographic RG flows as in the previous section.

A solution driving the flow from $E^{2,1} \times C Y 4$ to $A d S_{4} \times Q^{1,1,1}$

Since in this case there is an additional degree of freedom, a suitable change of variable, as compared to Eq.(16), is

$$
d r=e^{\phi+4 \lambda} d \tau
$$

A solution of the system (32)-(35) is

$$
\begin{array}{ll}
h(\tau)=\frac{1}{4} \log \left(\frac{2^{8 / 3} \Lambda}{3}+A e^{3 \tau / 2}\right), & \phi(\tau)=-7 / 6 \log (2)+h(\tau), \\
f(\tau)=\frac{\tau}{2}-\frac{1}{4} \log \left(2^{8 / 3} \Lambda+3 A e^{3 \tau / 2}\right), & \lambda=\frac{1}{6} \log (2),
\end{array}
$$

where $A$ is an integration constant. The corresponding eleven-dimensional metric is given by

$$
d s_{11}^{2}=\frac{2^{7 / 9} \times 3^{1 / 6} \times e^{\tau}}{\left(3 A e^{3 \tau / 2}+2^{8 / 3} \Lambda\right)^{2 / 3}} d x_{1,2}^{2}
$$




$$
\begin{aligned}
& +\frac{1}{2^{2 / 9} \times 3^{1 / 3}}\left(3 A e^{3 \tau / 2}+2^{8 / 3} \Lambda\right)^{1 / 3} d \tau^{2}+\frac{2^{7 / 9}}{3^{1 / 3}}\left(3 A e^{3 \tau / 2}+2^{8 / 3} \Lambda\right)^{1 / 3} \times \\
& \left(d \Omega_{1}^{2}+d \Omega_{2}^{2}+d \Omega_{3}^{2}+\frac{1}{2}\left(d \psi+\cos \alpha d \beta-\cos \theta_{1} d \phi_{1}-\cos \theta_{2} d \phi_{2}\right)^{2}\right) .
\end{aligned}
$$

In flat indices the four-form field strength is $F_{x_{1} x_{2} x_{3} \tau}=\frac{2^{7 / 9} \times 3^{7 / 6}}{\left(3 A e^{3 \tau / 2}+2^{8 / 3} \Lambda\right)^{7 / 6}}$, The solution in the $H$ and $s$ variables defined in the previous section is shown in figure 3 .

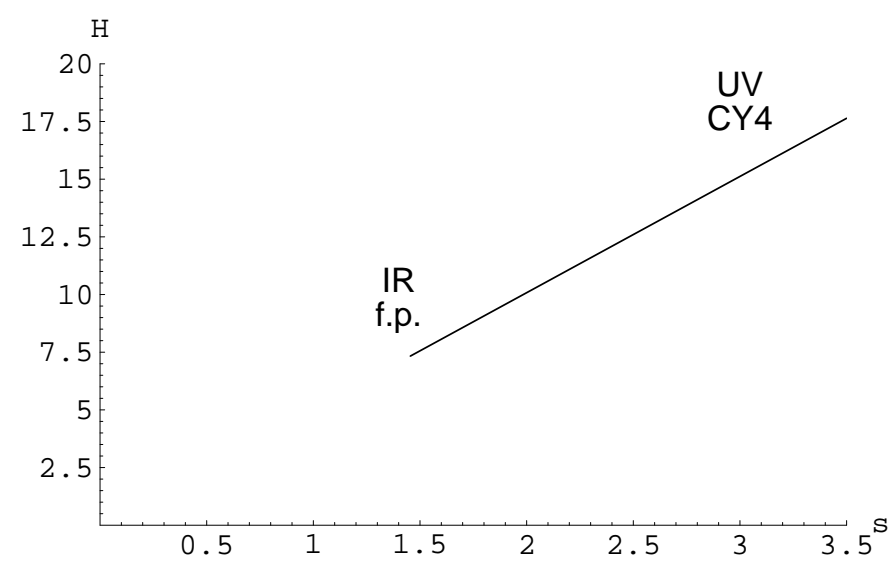

Figure 3: Solution as given by Eq.(39). The vertical axis is $H(s)$ and the horizontal one is $s$.

Using the change of variables given in Eq.(41) it is easy to see that the UV limit corresponds to $\tau \rightarrow+\infty$, while the IR one is for $\tau \rightarrow-\infty$. Therefore, let us first consider the limit $\tau \rightarrow \infty$. In M-theory, in the UV limit $(\tau \rightarrow+\infty)$, the system is turning off the $F_{4}$ field, such that the eleven-dimensional configuration must be pure metric. The number of preserved supercharges will be 4 and the metric will have an expression of the form $E^{2,1} \times C Y 4$, i.e. three flat dimensions plus a non-compact CY4 fold. This metric reads of

$$
\begin{aligned}
d s_{11}^{2}= & d x_{1,2}^{2}+d \rho^{2}+ \\
& \frac{\rho^{2}}{8}\left(d \Omega_{1}^{2}+d \Omega_{2}^{2}+d \Omega_{3}^{2}+\frac{1}{2}\left(d \psi+\cos \alpha d \beta-\cos \theta_{1} d \phi_{1}-\cos \theta_{2} d \phi_{2}\right)^{2}\right) .
\end{aligned}
$$

It is singular at $\rho=0$. As discussed above resolution of this singularity is achieved by flowing towards IR where $F_{4}$ is dynamically turned on. Hence, one should consider the complete metric (43). This metric is the M-theory dual of $\mathcal{N}=2 \mathrm{SYM}$ theory in three dimensions. In three dimensions the $\mathcal{N}=2$ supersymmetric algebra is the reduction of the

\footnotetext{
${ }^{5}$ We rescaled the variable $\frac{e^{\tau / 4}}{2^{1 / 9}} d \tau=d \rho$.
} 
$\mathcal{N}=1$ in four dimensions. The role of the central charge is played by the four component of the momentum. As in the higher dimensional case, the R-symmetry group is $U(1)_{R}$.

As usual, scalars in the vector superfield parameterize the Coulomb branch of the theory. In this case some fundamental hypermultiplets exist in the Lagrangian which will describe the Higgs branch. In our case, we expect a dynamical scalar field coming from fluctuations of the metric. Another way of understanding the presence of this dynamical scalar is by noticing that D6-branes wrap an $S^{2} \times S^{2}$ cycle inside a complex three-dimensional CY space. Thus, there will be one free direction (codimension one) in contrast to the $G_{2}$ case analyzed in the previous section. This direction is interpreted as a scalar field describing the Coulomb branch of the theory.

In addition, due to the presence of D2-branes, or due to the $C_{3}$ field in M-theory, some other scalar modes will have dynamics on the $2+1$-dimensional worldvolume.

In a non-Abelian theory, when we move into the Coulomb branch, we can dualize the vectors to scalars in linear multiples $A_{\mu} \rightarrow \gamma$ and the Coulomb branch is parametrized by the complex scalar $\Phi=\varphi+i \gamma$, like in four dimensions, this is the factor that appears when the instanton contributions are taken into account. In the case in which we have fundamental hypermultiplets, the interaction term is typically of the form

$$
V \sim \int d^{2} \theta d^{2} \bar{\theta} \bar{Q} e^{V} Q
$$

So, a bosonic term will be of the form $\bar{q} \varphi q$, this means that in general, the Coulomb branch and the Higgs branch will be disconnected. Nevertheless, there are situations in which we have a mixing of Coulomb and Higgs branches. We will not discuss these cases here, since our configurations will not have hypermultiplets. As it was studied by Affleck, Harvey and Witten, the instantons that are associated with $\Pi_{2}(G)$ are only present when we have a nonAbelian gauge group and we consider the Coulomb branch, so $\Pi_{2}\left(G / U(1)^{r}\right)=Z^{r}$. These instantons generate a superpotential that in the large $N$ limit goes to $W \sim e^{-N}$, so we cannot see it in a supergravity approximation. This is the reason why a brane probe of our configuration will lead to a 2-dimensional flat Coulomb branch. It would be of much interest, to find dual gravity configurations to theories with fundamental hypermultiplets.

On the other limit of the flow, i.e. when $\tau \rightarrow-\infty$, one obtains the metric

$$
\begin{aligned}
d s_{11}^{2}= & e^{\xi \rho} d x_{1,2}^{2}+d \rho^{2}+2\left(\frac{4|\Lambda|}{3}\right)^{1 / 3} \times \\
& \left(d \Omega_{1}^{2}+d \Omega_{2}^{2}+d \Omega_{3}^{2}+\frac{1}{2}\left(d \psi+\cos \alpha d \beta-\cos \theta_{1} d \phi_{1}-\cos \theta_{2} d \phi_{2}\right)^{2}\right),
\end{aligned}
$$

where as before, $d \Omega_{i}^{2}$ denotes the line element over a two-sphere ${ }^{\top}$. In addition, one has a four-form field of the form $F_{x_{1} x_{2} x_{3} \tau}=\frac{2^{7 / 9} 3^{7 / 6}}{\left(2^{8 / 3} \Lambda\right)^{7 / 6}}$. The manifold in Eq. (46) is $A d S_{4} \times Q^{1,1,1}$ and

\footnotetext{
${ }^{6}$ We rescaled the variable $\tau$ as $\frac{2^{1 / 3} \Lambda^{1 / 6}}{3^{1 / 6}} d \tau=d \rho$.
} 
the conformal field theory to which this manifold is dual is well-known. Indeed, the manifold $Q^{1,1,1}$ has been well studied in the past. The isometries of this space are $S U(2)^{3} \times U(1)$ and are in correspondence with the global symmetries of the CFT. The KK modes on $Q^{1,1,1}$ were worked out in [86. It contains short and long multiplets of $O s p(2 \mid 4)$.

By linearizing the BPS equations (33-35) near the fixed point, one can work out the scale dimensions of the operators dual to the eigenvectors of the mass matrix which are combinations of $\phi, h$ and $\lambda$. Dimensions turn out to be,

$$
\Delta_{1}=6, \quad \Delta_{2,3}=\frac{3}{2} \pm \sqrt{\frac{31}{12}} .
$$

Note, however, that in Eq.(42) latter two of the eigenvectors are turned off by requiring $\lambda=$ const. and $h-\phi=$ const.. Thus, as in the previous example, one is left with an irrelevant "overall size" deformation of $Q^{1,1,1}$ with dimension $\Delta=6$ and mass, $M^{2}=72$. Therefore the dual CFT is at IR. Solutions obtained by turning on more eigenvectors are presented in Appendix B, for which AdS geometry appears at UV, as one expects. KK spectrum of $A d S_{4} \times Q^{1,1,1}$ compactification was partially worked out in 87 and one indeed finds that the "overall size" deformation is a singlet of the bosonic isometry $S U(2) \times S U(2) \times S U(2)$ and $U(1) R$-symmetry together with (in our normalization conventions) $\Delta=6$ and mass, $M^{2}=72$.

The CFT dual to the metric (46) corresponds to the one in an M2-brane on the tip of a cone on the seven-dimensional manifold 88, 89, 90, 91, 92, 93, 94, 95. This gauge theory has a moduli space of vacua isomorphic to $Q^{1,1,1}$.

Like other CFT's the theory has a Coulomb branch described by fields in the vector multiplet and a Higgs branch described by fields in chiral multiplets. Working out the theory whose Higgs branch is dual to the conifold above, one finds that fundamental fields are doublets with respect to the flavour group $S U(2)^{3}: A_{i}, B_{i}, C_{i}$ with $i=1$, 2 , i.e., the fields transform as $A_{i}=(2,1,1), B_{i}=(1,2,1), C_{i}=(1,1,2)$ under the flavour group. The gauge theory has the color symmetry $S U(N) \times S U(N) \times S U(N)$, with elementary degrees of freedom transforming in the fundamental and anti-fundamental representation of the $S U(N)^{\prime} s$, namely $A_{i}=(N, \bar{N}, 1), B_{i}=(1, N, \bar{N}), C_{i}=(\bar{N}, 1, N)$. These fields have conformal weight $c=1 / 3$, hence one can construct gauge invariant operators of the form

$$
X^{i j k}=A^{i} B^{j} C^{k}
$$

out of them. These eight operators are singlets under the global symmetries and have conformal weight equal to one.

One important point is the comparison of the $\mathrm{KK}$ modes on the $Q^{1,1,1}$ manifold and the spectrum of hypermultiplets in the CFT. The spectrum of the Laplacian in $Q^{1,1,1}$ is computed and one can associate it with a chiral multiplet in the $(k / 2, k / 2, k / 2)$ representation of $S U(2)^{3}$ with dimension $E=k$. Therefore, it is natural to make a correspondence with composite 
operators of the form $\operatorname{Tr}(A B C)^{k}$ with symmetrized $S U(2)$ indices. This agrees with the gauge theory.

Nevertheless, there are some operators in gauge theory-like those where the $S U(2)$ indices are not symmetrized-that do not have a KK analog. One would think that a superpotential can be generated in such a way to get rid of those states as in the case of $T^{1,1}$, but this is not the case. Indeed, we can see from a gauge theory perspective that the potential that should do the job

$$
\begin{aligned}
V \sim & {\left[\left(\left|A_{1}\right|^{2}+\left|A_{2}\right|^{2}-\left|C_{1}\right|^{2}-\left|C_{2}\right|^{2}\right)^{2}+\left(\left|B_{1}\right|^{2}+\left|B_{2}\right|^{2}-\left|A_{1}\right|^{2}-\left|A_{2}\right|^{2}+\right)^{2}\right.} \\
& \left.+\left(\left|C_{1}\right|^{2}+\left|C_{2}\right|^{2}-\left|B_{1}\right|^{2}-\left|B_{2}\right|^{2}\right)^{2}\right]
\end{aligned}
$$

vanishes due to the fact that it is exactly the (Higgs branch) description of the manifold $Q^{1,1,1}$. So, the potential does not solve the problem and one needs to assume that these unwanted colored degrees of freedom are not chiral primaries.

One can also find the presence of a baryonic operator essentially corresponding to wrapping an M5-brane on a five-cycle inside the eight-cone. The operators corresponding to baryons are of the form $\operatorname{det}[A], \operatorname{det}[B], \operatorname{det}[C]$. Since our manifold $Q^{1,1,1}$ has Betti numbers $b_{2}, b_{5} \operatorname{different}$ from zero, there is another $U(1)$ under which only non-perturbative states will be charged. In our case, the baryonic symmetry acts on the fundamental fields as $A_{i}=(1,-1,0), B_{i}=$ $(0,1,-1), C_{i}=(-1,0,1)$, therefore we can see that gauge invariant operators $X$ are not charged under baryon number. One can compute the dimension of the baryonic operator by computing the mass of an M5-brane wrapping a five-cycle inside the cone. This mass in the case of a supersymmetric cycle, coincides with the volume of the cycle. In our case the five-cycle is a $U(1)$ fibre over $S^{2} \times S^{2}$ and since our manifold is a $U(1) \rightarrow S^{2} \times S^{2} \times S^{2}$ we have three different supersymmetric cycles that are associated with the three operators defined above. Each cycle is supersymmetric as we can see from the twisting condition described above. The volume of the cycle, can be computed to be proportional to $N / 3$ thus confirming the fact that each operator $A, B, C$ have dimension $1 / 3$. If the M5-brane wraps a three-cycle, the object is interpreted as a domain wall of the CFT.

\subsection{The case of $\mathcal{N}=3$ supersymmetry: from $\mathrm{HK}$ to $N^{0,1,0}$}

Here, we will briefly comment on the case where the D6-branes are wrapping a four-cycle that preserves $\mathcal{N}=3$ supersymmetry. The set up is very similar to the previous examples, except we take the four-cycle to be a $C P^{2}$ manifold. We choose a metric (using as coordinates $\xi$ and the three angles in the left-invariant forms $\sigma^{i}$ )

$$
d s_{C P 2}^{2}=d \xi^{2}+\frac{1}{4} \sin ^{2} \xi\left(\sigma_{1}^{2}+\sigma_{2}^{2}+\cos ^{2} \xi \sigma_{3}^{2}\right) .
$$


The gauge field which provides the twisting preserving six supercharges is given by

$$
A^{(i)}=\cos \xi \sigma^{(i)}, \quad A^{(3)}=\frac{1}{2}\left(1+\cos ^{2} \xi\right) \sigma^{(3)} .
$$

A solution of the BPS equations lifted to eleven dimensions reads

$$
d s_{11}^{2}=\frac{d x_{1,2}^{2}}{\left(1+\frac{B}{r^{6}}\right)^{2 / 3}}+\left(r^{6}+B\right)^{1 / 3} d s_{C P 2}^{2}+2\left(\frac{B}{r^{6}}+1\right)^{1 / 3} d r^{2}+\frac{r^{2}}{2}\left(\frac{B}{r^{6}}+1\right)^{1 / 3}\left(\omega^{i}-A^{i}\right)^{2}
$$

together with the four-form field strength

$$
F_{x y z r}=\frac{3 B}{\left(B+r^{6}\right)^{7 / 6}},
$$

written in flat indices, where $B$ is a constant. Therefore, the metric (52) represents a holographic RG flow from $E^{2,1} \times H K$ (hyperKahler) at UV to $A d S_{4} \times N^{0,1,0}$ at IR. The isometry group of $N^{0,1,0}$ is $S U(3) \times S U(2)$ while its holonomy is $S U(2)$.

As it is known, an $\mathcal{N}=3$ supersymmetric gauge theory has the field content of an $\mathcal{N}=4$ supersymmetric gauge theory, plus an interaction that respects three out of the four spinors.

It was shown by Kapustin and Strassler [96] that for the Abelian case, the ways of breaking $\mathcal{N}=4$ down to $\mathcal{N}=3$ supersymmetry are either by adding a Chern Simons term or with a mass term for a chiral superfield $Y^{I}$ in the adjoint representation of the gauge group.

In our case, we have a supergravity solution of the form $A d S_{4} \times N^{0,1,0}$. The dual gauge theory in the IR limit will have a gauge group $S U(N) \times S U(N)$ and a flavor group $S U(3)$. There will be two hypermultiplets, $u_{1}, u_{2}$ and $v_{1}, v_{2}$ transforming in the $(3, N, \bar{N})$ and $(\overline{3}, \bar{N}, N)$ representations and two chiral multiplets, $Y_{(1)}, Y_{(2)}$ in the adjoint representation of $S U(N)$. There is a superpotential of the form

$$
V \sim g_{i} \operatorname{Tr}\left(Y_{(i)} \vec{u} \cdot \vec{v}\right)+\alpha_{i} \operatorname{Tr}\left(Y_{(i)} Y_{(i)}\right),
$$

where $g_{i}$ are the gauge couplings of each $S U(N)$ group and $\alpha_{i}$ are the Chern Simons coefficients. Interesting aspects of this theory, like the KK spectrum of the compactifications and different checks of the duality have been studied in [97, 98].

\section{Penrose limits and pp-waves}

In this section we will show how to obtain the pp-waves in the Penrose limit for the IR region of the supergravity solutions described in sections 4 and 5 .

We will focus on the solutions with $\mathcal{N}=2$ and $\mathcal{N}=3$ supersymmetry and we will add a brief description of the $\mathcal{N}=1$ case near the end of the section. 
The interest of taking the Penrose limit is based on the fact that it could be possible to define following [37] a Matrix model to check the correlation between the gravity and the gauge theory side. We postpone the checks of this correlations for a future publication, here we will only concentrate on the gravity aspects.

Penrose limit of the AdS $\times$ Einstein-Sasakian manifold

The Einstein metric of $A d S_{4} \times Q^{1,1,1}$ can be written as

$$
d s_{11}^{2}=d s_{A d S_{4}}^{2}+d s_{Q^{1,1,1}}^{2},
$$

where

$$
d s_{A d S_{4}}^{2}=R^{2}\left(-d t^{2} \cosh ^{2} \rho+d \rho^{2}+\sinh ^{2} \rho d \Omega_{2}^{2}\right),
$$

and

$$
\begin{aligned}
d s_{Q^{1,1,1}}^{2}= & \mu^{2} R^{2}\left(d \theta_{1}^{2}+\sin ^{2} \theta_{1} d \phi_{1}^{2}+d \theta_{2}^{2}+\sin ^{2} \theta_{2} d \phi_{2}^{2}+d \theta_{3}^{2}+\sin ^{2} \theta_{3} d \phi_{3}^{2}+\right. \\
& \left.\frac{1}{2}\left(d \psi+\cos \theta_{1} d \phi_{1}+\cos \theta_{2} d \phi_{2}+\cos \theta_{3} d \phi_{3}\right)^{2}\right) .
\end{aligned}
$$

Where $\mu$ is the relation between the radii of $A d S_{4}$ and $Q^{1,1,1}$. Topologically $Q^{1,1,1}$ is a $U(1)$ bundle over $S^{2} \times S^{2} \times S^{2}$, so that it can be parametrized by $\left(\theta_{1}, \phi_{1}\right),\left(\theta_{2}, \phi_{2}\right)$ and $\left(\theta_{3}, \phi_{3}\right)$ coordinates over each $S^{2}$, respectively, while the period of the Hopf fiber coordinate $\psi$ is $4 \pi$. The $S U(2)_{1} \times S U(2)_{2} \times S U(2)_{3} \times U(1)$ isometry of $Q^{1,1,1}$ is identified with the $S U(2)_{1} \times S U(2)_{2} \times S U(2)_{3}$ global symmetry and $U(1)_{R}$ symmetry of the dual $S U(N) \mathcal{N}=2$ SCFT in three dimensions.

Now, the idea is to obtain a certain scaling limit around a null geodesic in $A d S_{4} \times Q^{1,1,1}$. This rotates the $\psi$ coordinate of $Q^{1,1,1}$ in correspondence with the $U(1)_{R}$ symmetry of the dual SCFT. Moreover, the changes in the angles $\phi_{1}, \phi_{2}$ and $\phi_{3}$ generate an $U(1)_{1} \times U(1)_{2} \times$ $U(1)_{3} \subset S U(2)_{1} \times S U(2)_{2} \times S U(2)_{3}$ isometry. In the SCFT side this is generated by the dual Abelian charges $Q_{1}, Q_{2}$ and $Q_{3}$, which are the Cartan generators of the global $S U(2)_{1} \times S U(2)_{2} \times S U(2)_{3}$ symmetry group of the field theory.

Thus we define new coordinates

$$
\begin{aligned}
& x^{+}=\frac{1}{2}\left(t+\frac{\mu}{\sqrt{2}}\left(\psi+\phi_{1}+\phi_{2}+\phi_{3}\right)\right), \\
& x^{-}=\frac{R^{2}}{2}\left(t-\frac{\mu}{\sqrt{2}}\left(\psi+\phi_{1}+\phi_{2}+\phi_{3}\right)\right) .
\end{aligned}
$$

Note the scaling in the latter equation by $R^{2}$. We will consider a scaling limit around $\rho=\theta_{1}=\theta_{2}=\theta_{3}=0$ in the metric above, such that when we take the limit $R \rightarrow \infty$ we also scale the coordinates as follows

$$
\rho=\frac{r}{R}, \quad \theta_{1}=\frac{\zeta_{1}}{R}, \quad \theta_{2}=\frac{\zeta_{2}}{R}, \quad \theta_{3}=\frac{\zeta_{2}}{R} .
$$


Therefore, the Penrose limit of the $A d S_{4} \times Q^{1,1,1}$ metric is given by $d s_{11}^{2}=-4 d x^{+} d x^{-}+\sum_{i=1}^{3}\left(d r^{i} d r^{i}-r^{i} r^{i} d x^{+} d x^{+}\right)+\sum_{i=1}^{3}\left(\mu^{2} d \zeta_{i}^{2}+\mu^{2} \zeta_{i}^{2} d \phi_{i}^{2}-\frac{\mu}{\sqrt{2}} \zeta_{i}^{2} d \phi_{i} d x^{+}\right)$.

Changing to the complex coordinates $z_{j}=\zeta_{j} e^{i \phi_{j}}$ one obtains

$d s_{11}^{2}=-4 d x^{+} d x^{-}+\sum_{i=1}^{3}\left(d r^{i} d r^{i}-r^{i} r^{i} d x^{+} d x^{+}\right)+\sum_{j=1}^{3}\left(\mu^{2} d z_{j} d \bar{z}_{j}+i \frac{\mu}{2 \sqrt{2}}\left(\bar{z}_{j} d z_{j}-z_{j} d \bar{z}_{j}\right) d x^{+}\right)$.

This metric has a covariantly constant null Killing vector $\partial / \partial x^{-}$, and therefore is a ppwave metric having a decomposition of $\mathbf{R}^{9}$ as $\mathbf{R}^{3} \times \mathbf{R}^{2} \times \mathbf{R}^{2} \times \mathbf{R}^{2}$. Three-dimensional Euclidean space is parametrized by $r_{i}$, while $\mathbf{R}^{2} \times \mathbf{R}^{2} \times \mathbf{R}^{2}$ is parametrized by $z_{j}$ above. In addition, the background has a constant $F_{+x_{1} x_{2} r}$. The symmetries of this configuration are the $S O(3)$ rotations in $\mathbf{R}^{3}$ and $U(1) \times U(1) \times U(1)$ symmetry related to the $\mathbf{R}^{2} \times \mathbf{R}^{2} \times \mathbf{R}^{2}$ rotations. We choose this particular Penrose limit due to the fact that these $U(1)$ 's are representing the symmetries of the gauge theory dual to this background. From the dual field theory viewpoint, the $S O(3)$ isometry is a subgroup of the $S O(2,3)$ conformal group. $U(1) \times U(1) \times U(1)$ rotational charges $J_{1}, J_{2}$ and $J_{3}$ correspond to differences between $U(1) \times U(1) \times U(1)$ charges $Q_{1}, Q_{2}$ and $Q_{3}$ and the $U(1)_{R}$ charge. Indeed from the field theory side, it is expected to deal with operators with large $U(1)_{R}$ symmetry charge $J$, being the charges $Q_{1}, Q_{2}$ and $Q_{3}$ also scaled as $\sqrt{N}$ like $J$, while the corresponding rotational charges $J_{i}$ 's would remain finite.

After an $U(1) \times U(1) \times U(1)$ rotation in the $\mathbf{R}^{2} \times \mathbf{R}^{2} \times \mathbf{R}^{2}$ plane as

$$
z_{j}=e^{i \sqrt{2} x^{+} /(4 \mu)} w_{j}, \quad \bar{z}_{j}=e^{-i \sqrt{2} x^{+} /(4 \mu)} \bar{w}_{j},
$$

one obtains a metric that after suitable rescalings of its variables turns out to be

$$
d s_{11}^{2}=-4 d x^{+} d x^{-}-\left(\left(\frac{\mu}{6}\right)^{2} \vec{r}_{3}^{2}+\left(\frac{\mu}{3}\right)^{2} \vec{y}_{6}^{2}\right) d x^{+} d x^{+}+d \vec{r}_{3}^{2}+d \vec{y}_{6}^{2},
$$

where $y_{j}$ are the 6 coordinates on $\mathbf{R}^{2} \times \mathbf{R}^{2} \times \mathbf{R}^{2}$. The above metric corresponds to the maximally supersymmetric pp-wave solution of $A d S_{4} \times S^{7}$. It means that the dual SCFT is $\mathcal{N}=8, S U(N)$ super Yang Mills theory in three dimensions. This shows the enhancement of supersymmetry analogous to the ones obtained in 41, 42, 44. This fact might be interpreted as a hidden $\mathcal{N}=8$ supersymmetry which was already present in the corresponding subsector of the dual $\mathcal{N}=2 \mathrm{SCFT}$.

Penrose limit of the $A d S \times N^{0,1,0}$ manifold

The Einstein metric of $A d S_{4} \times N^{0,1,0}$ can be written as

$$
d s_{11}^{2}=d s_{A d S_{4}}^{2}+d s_{N^{0,1,0}}^{2}
$$


where we again use

$$
d s_{A d S_{4}}^{2}=R^{2}\left(-d t^{2} \cosh ^{2} \rho+d \rho^{2}+\sinh ^{2} \rho d \Omega_{2}^{2}\right)
$$

while

$$
\begin{aligned}
d s_{N, 1,0}^{2}= & \mu^{2} R^{2}\left(d \zeta^{2}+\frac{\sin ^{2} \zeta}{4}\left(d \theta^{2}+\sin ^{2} \theta d \phi^{2}+\cos ^{2} \zeta(d \psi+\cos \theta d \phi)^{2}\right)+\right. \\
& \frac{1}{2}(\cos \gamma d \alpha+\sin \gamma \sin \alpha d \beta-\cos \zeta(\cos \psi d \theta+\sin \psi \sin \theta d \phi))^{2}+ \\
& \frac{1}{2}(-\sin \gamma d \alpha+\cos \gamma \sin \alpha d \beta-\cos \zeta(-\sin \psi d \theta+\cos \psi \sin \theta d \phi))^{2}+ \\
& \left.\frac{1}{2}\left(d \gamma+\cos \alpha d \beta-\frac{1}{2}\left(1+\cos ^{2} \zeta\right)(d \psi+\cos \theta d \phi)\right)^{2}\right) .
\end{aligned}
$$

Where $\mu$ is the relation between the $A d S_{4}$ and $N^{0,1,0}$ radii. Again, the idea is to obtain a certain scaling limit around a null geodesic in $A d S_{4} \times N^{0,1,0}$. In this case we can define the coordinates

$$
\begin{aligned}
& x^{+}=\frac{1}{2}\left(t+\frac{\mu}{\sqrt{2}}(\gamma+\beta-\psi / 2-\phi / 2)\right), \\
& x^{-}=\frac{R^{2}}{2}\left(t-\frac{\mu}{\sqrt{2}}(\gamma+\beta-\psi / 2-\phi / 2)\right) .
\end{aligned}
$$

We will consider a scaling limit around $\rho=\theta=\alpha=0$ and $\zeta=\pi / 2$ in the metric above, so that when we take the limit $R \rightarrow \infty$ we also scale the coordinates as

$$
\rho=\frac{r}{R}, \quad \zeta=\frac{\pi}{2}+\frac{x}{R}, \quad \alpha=\frac{z}{R}, \quad \theta=\frac{y}{R} .
$$

Therefore, after some appropriate redefinitions of coordinates, the Penrose limit of the $A d S_{4} \times$ $N^{0,1,0}$ metric is given by

$$
\begin{aligned}
d s_{11}^{2}= & -4 d x^{+} d x^{-}+\sum_{i=1}^{3}\left(d r^{i} d r^{i}-r^{i} r^{i} d x^{+} d x^{+}\right)+\mu^{2}\left(z^{2} d \beta^{2}+y^{2} d \phi^{2}+x^{2} d \hat{\psi}^{2}\right)+ \\
& \mu^{2}\left(d x^{2}+d y^{2}+d z^{2}\right)-\sqrt{2} \mu d x^{+}\left(z^{2} d \beta+y^{2} d \phi+x^{2} d \hat{\psi}\right)
\end{aligned}
$$

where we have changed $\psi+\phi \rightarrow \hat{\psi}$.

Using the redefinitions

$$
x=\zeta_{1}, \quad y=\zeta_{2}, \quad z=\zeta_{3},
$$

and

$$
\hat{\psi}=\phi_{1}, \quad \phi=\phi_{2}, \quad \beta=\phi_{3},
$$

together with a further rescaling, this metric becomes exactly Eq. 61). Therefore, we can follow a similar path defining complex coordinates, etc., obtaining that the Penrose limit of 
$A d S_{4} \times N^{0,1,0}$ reduces to the same pp-wave as the corresponding one of $A d S_{4} \times S^{7}$. Hence, we might conclude that likely there is a hidden $\mathcal{N}=8$ supersymmetry which was already present in the corresponding subsector of the dual $\mathcal{N}=3 \mathrm{SCFT}$.

\section{Penrose limit of the $A d S \times$ squashed seven-sphere}

Finally, we would like to add some comments on the case of the Spin(7) holonomy manifold, that is our solution preserving $\mathcal{N}=1$ supersymmetry. This case is analogous to the Penrose limit for the gravity solution of D5-branes on the resolved conifold, that has been worked out in 42$]$ ?.

The Einstein metric of $A d S_{4} \times \tilde{S}^{7}$ can be written as

$$
d s_{11}^{2}=d s_{A d S_{4}}^{2}+d s_{\tilde{S}^{7}}^{2}
$$

where as before we have

$$
d s_{A d S_{4}}^{2}=R^{2}\left(-d t^{2} \cosh ^{2} \rho+d \rho^{2}+\sinh ^{2} \rho d \Omega_{2}^{2}\right),
$$

while

$$
d s_{\tilde{S}^{7}}^{2}=\mu^{2}\left(d \Omega_{4}^{2}+\frac{1}{5}\left(\omega^{i}-A^{i}\right)\right),
$$

where $\mu$ stands for the relation between AdS and squashed seven-sphere radii, and the factor $1 / 5$ comes from the relation between $S^{4}$ and $S U(2)$-group manifold radii. We remind that the isometries of $\tilde{S}^{7}$ are $S O(5) \times S U(2)$.

Indeed, we can proceed in the following way, we rescale the coordinates such that the part coming from the four-sphere reads

$$
d \Omega_{4}^{2} \approx \frac{d \tau^{2}}{R^{2}}+\frac{\tau^{2}}{4 R^{2}} d \Omega_{3}^{2}
$$

that is an $\mathbf{R}^{4}$ space. This rescaling includes $\rho \rightarrow r / R, \theta \rightarrow y / R$ and $\alpha \rightarrow z / R$, where the angles are used to define the left-invariant one forms and the precise definition is given in Appendix D.

In this coordinates the gauge field will be approximated by $A^{i} \approx\left(1-\frac{\tau^{2}}{R^{2}}\right) \sigma^{i}$. In the limit of large $R$, the term in the metric describing the fibration between the coordinates of the three-sphere and the four-sphere will basically consist of two parts. After a suitable change of variables, the term coming from $\left(\omega^{i}-\sigma^{i}\right)^{2}$ will contribute to $\left(d x^{+}-d x^{-} / R^{2}\right)^{2}$ and a flat two-dimensional space. The second term in the metric (77) proportional to $\tau$ will contribute with a term of the form $\tau^{2} / R^{2} d x^{+} d \phi$. After a similar rescaling as the one for the $\mathcal{N}=2$ case is done, it will add a mass term for two of the flat directions, and we obtain a metric that looks very similar to Eq.(61).

\footnotetext{
${ }^{7}$ We thank Jaume Gomis for explanations on this respect.
} 


\section{RG flows from gauged supergravity in 6 dimensions}

In this section we will present other Holographic RG flow examples obtained from $F(4)$ gauged supergravity 99 in 6 dimensions. These will be of interest since uplifting of these solutions to massive IIA supergravity is known [52]. The bosonic Lagrangian is

$$
e^{-1} \mathcal{L}_{B}^{(6)}=-\frac{1}{4} R+\left(\partial^{\mu} \varphi\right)\left(\partial_{\mu} \varphi\right)-V(\varphi)
$$

where we set the Abelian, non-Abelian and the two-index tensor gauge fields to zero. The dilaton potential is given by

$$
V(\varphi)=-\frac{1}{8}\left(g^{2} e^{2 \varphi}+4 m g e^{-2 \varphi}-m^{2} e^{-6 \varphi}\right)
$$

where $g$ is the non-Abelian coupling constant and $m$ becomes the mass of $B_{\mu \nu}$ field via Higgs mechanism 99]. Figure 4 shows the dilaton potential. Without loss of generality one can set $g=3 m$ which will yield a supersymmetric background at the maximum of $V$ at $\varphi=0$. At the two extrema of the potential,

$$
\varphi_{\min }=-\frac{1}{4} \log (3), \quad V\left(\varphi_{\min }\right)=-\frac{3 \sqrt{3}}{2} m^{2}
$$

and,

$$
\varphi_{M a x}=0, \quad V\left(\varphi_{M a x}\right)=-\frac{5}{2} m^{2} .
$$

which correspond to $A d S_{6}$ solutions with curvatures $R_{\text {Min }}=9 \sqrt{3} \mathrm{~m}^{2}$ and $R_{\text {Max }}=15 \mathrm{~m}^{2}$, respectively. The Euler-Lagrange equations are

$$
\begin{aligned}
& 0=R_{\mu \nu}-4 \partial_{\mu} \varphi \partial_{\nu} \varphi+g_{\mu \nu} V(\varphi) \\
& 0=-2 \square \varphi-\frac{\partial V}{\partial \varphi} .
\end{aligned}
$$

These fixed-point solutions can be lifted to massive type IIA string theory using the uplifting procedure given by [52] where the Romans' $\mathrm{F}(4)$ gauged supergravity in 6 dimensions is obtained from a consistent warped $S^{4}$ reduction of massive type IIA string theory. 


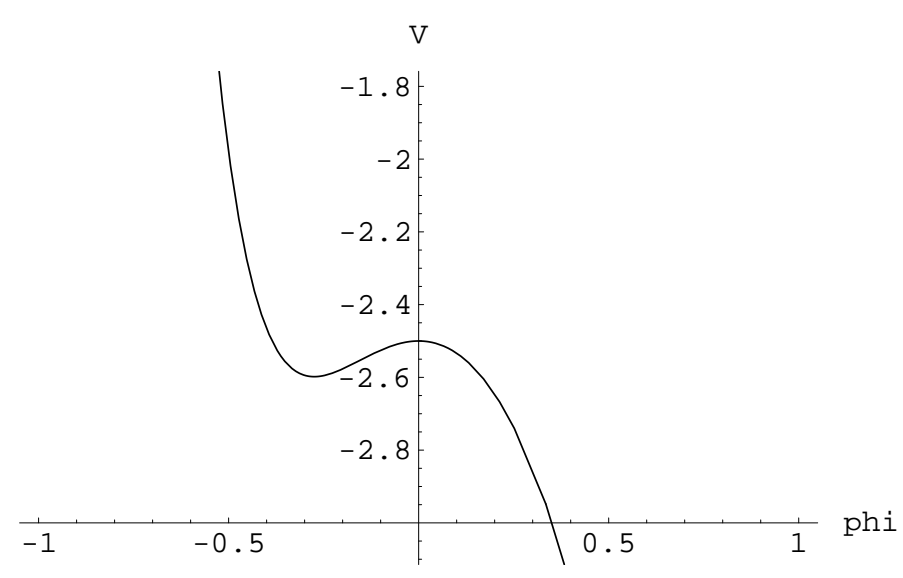

Figure 4: Scalar potential for 6-dimensional gauged supergravity in units $m^{2}=1$.

\section{$7.1 \quad$ Interpolating Solutions}

Non-supersymmetric flow

One can obtain a kink solution to EOM which interpolates between local maximum and minimum of $V(\phi)$. We make the usual domain-wall ansatz,

$$
d s^{2}=e^{2 f(r)} \eta_{\mu \nu} d x^{\mu} x^{\nu}-d r^{2}
$$

with mostly minus convention. For $f(r)=r / l$ the metric becomes $A d S_{6}$ with $l$ constant. The kink is the general solution $f(r)$ interpolating between two $A d S_{6}$ spacetimes with different radius $l$. In these coordinates the UV limit is given when $f \rightarrow+\infty$, while the IR limit corresponds to $f \rightarrow-\infty$. One obtains the following EOM from Eq.(82) and Eq.(83).

$$
\begin{aligned}
A^{\prime \prime} & =-\left(\varphi^{\prime}\right)^{2} \\
5 A^{\prime} \varphi^{\prime}+\varphi^{\prime \prime} & =+\frac{1}{16} \frac{\partial V(\varphi)}{\partial \varphi}
\end{aligned}
$$

with the following boundary conditions

$$
\left.\varphi^{\prime}\right|_{\phi=0}=\left.\varphi^{\prime}\right|_{\phi=-\frac{1}{4}}=0
$$

It is easy to see that a superpotential $W(\phi)$ defined by,

$$
-V(\varphi)=5 W(\varphi)^{2}-\left(\frac{\partial W}{\partial \varphi}\right)^{2}
$$


exists but does not have two extrema. Hence the kink interpolating between two $A d S_{6}$ solutions will necessarily be non-supersymmetric. In this case, one has to solve the second order EOM given above. The solution corresponds to flowing towards left from the origin in figure 4. We could not find an analytic solution mostly due to the lack of supersymmetry along the flow, however a numerical solution can be obtained. A similar study of an RG flow from seven-supergravity was done in [101]. Figure 5 shows the interpolating solution.

In order to identify the dual field theories at both UV and IR ends of the flows, one solves the scalar equation of motion linearized near the extrema. The solution near the UV fixed point reads,

$$
\varphi=A_{1} e^{-2 r}+A_{2} e^{-3 r} .
$$

Noting that $\varphi<0$ along the flow, we see that any VEV type deformation is excluded, otherwise VEV's of the operators would be negative which is not physical. Therefore, the deformation is a source term with scale dimension $\Delta_{1}=3$ or $\Delta_{2}=2$. The second case is also excluded since there does not exist any bosonic operator in five-dimensional CFT of dimension 2, hence we conclude that the RG flow is initiated by deforming the CFT with a mass term to the chiral superfield,

$$
\int d^{5} x \operatorname{Tr}[\Phi \Phi],
$$

with the scale dimension $\Delta_{1}=3$. In Eq.(89) this corresponds to setting $A_{2}=0$.

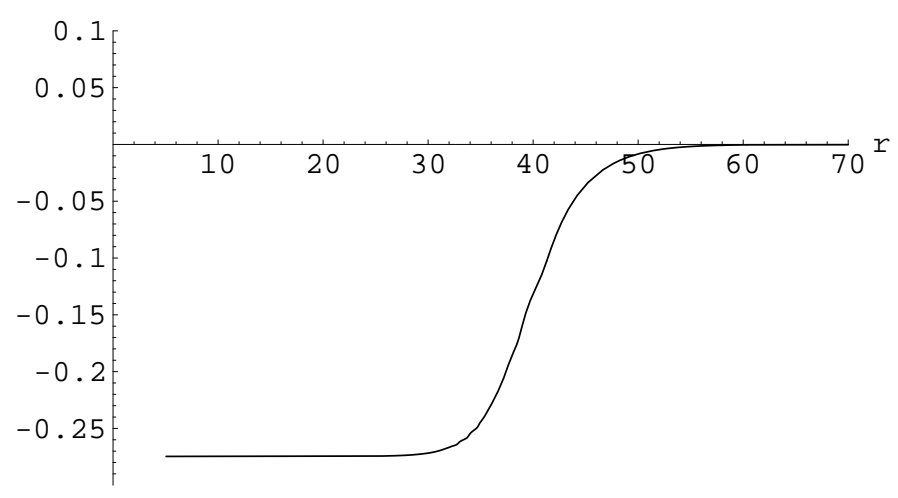

Figure 5: $\varphi$ as a function of $r$.

Linearization near IR fixed point yields scale dimensions,

$$
\Delta_{1,2}=\frac{1}{2}(5 \pm \sqrt{65})
$$


which shows that the source term acquires an anomalous dimension. This is of course expected in the absence of symmetries which would protect the scale dimension if the operator.

\section{Supersymmetric flow}

Another possible kink solution is interpolating between the maximum at $\varphi=0$ to $\varphi=+\infty$. This corresponds to flowing from the origin towards right in figure 4 . Since there is a curvature singularity at $\phi=+\infty$, one has to decide whether the flow can be physical by means of the Gubser's criterion 85. Since the scalar potential is bounded from above in that limit we find that the curvature singularity is good type.

A superpotential is obtained from, Eq.(88), (up to a sign),

$$
W=\frac{1}{4}\left(3 e^{\varphi}+e^{-3 \varphi}\right) .
$$

Note that we are using limits in which $l$ is set to 1 . Accordingly, second order EOM are reduced to the first order Killing spinor equation,

$$
\frac{\partial \varphi}{\partial r}=\frac{3}{4}\left(e^{-3 \varphi}-e^{\varphi}\right)
$$

with the solution,

$$
r=\text { const }+\frac{1}{3}\left(2 \arctan \left(e^{-\varphi}\right)-\log \left(1-e^{-\varphi}\right)+\log \left(e^{-\varphi}+1\right)\right) .
$$

Although one can not invert this equation into the form $\varphi(r)$, it contains the same information. Note that in the UV limit $\varphi \rightarrow 0, r \rightarrow+\infty$ and in the IR limit $\varphi \rightarrow+\infty, r \rightarrow$ const as expected from an RG flow between conformal and non-conformal theories [100.

Expansion of $W(\varphi)$ near the UV fixed point leads to the linearized solution around the maximum,

$$
\varphi=A e^{-3 r}
$$

Since there can not be a bosonic source term of dimension 1 , we conclude that this is a pure Higgs type deformation [102 where the $\Delta=3$ operator, $m^{2} \operatorname{Tr}[\Phi \Phi]$ acquires a VEV. As a result, conformal supercharges are broken whereas Poincare supercharges are preserved, i.e. there are 8 supercharges along the flow. In the dual field theory, $R$-symmetry group $S O(4)$ is broken down to $S O(2) \times S O(2)$. This is the analog of Coulomb branch flow of $\mathcal{N}=4$ SYM [103].

\section{Final comments}

We would like to summarize the different points studied in this paper. We constructed a set of solutions describing D2-D6 brane system where the D6-branes wrap different supersymmetry 
four-cycles in several manifolds with special holonomy. In order to find these solution we have used the Salam-Sezgin eight-dimensional gauged supergravity. These solutions represent holographic RG flows for three-dimensional supersymmetric gauge field theories. We have analyzed some aspects of the dual gauge theories that turn out to be SCFT's preserving $\mathcal{N}=1,2$, and 3 supersymmetries. Since at large distances, the metrics look like a direct product of three-dimensional Minkowski spacetime times an eight manifold, we motivate our approach as a possible "resolution" of the eight-manifold singularity, by turning on the $F_{4}$ field.

We then studied the Penrose limits of the near horizon region in the metrics above, and arrived at a phenomenon that seems to be of general feature, namely, the pp-wave limit looks like the geometry that one would obtain by replacing the cone by a round sphere. One can think of it as the limit is "erasing" the details of the particular manifolds that we consider. This gives rise to an interesting supersymmetry enhancement phenomenon on the dual field theory which was already noted very recently in the particular case of $\mathcal{N}=1$ super Yang-mills in four dimensions. Hence, we might conclude that likely there is a hidden $\mathcal{N}=8$ supersymmetry which is present in the corresponding subsector of the dual SCFTs with less supersymmetries.

Finally, in an unrelated last section, we studied an RG flow between two $A d S_{6}$ spaces. One of them preserves supersymmetry and it corresponds to a D4-D8 system. The second $A d S_{6}$ space is non-supersymmetric. We have obtained numerically a kink solution interpolating between the two vacua and commented on some gauge theory aspects like the dimensions of the operators that are inserted.

We would like to mention some open problems discussed in the paper. It would be interesting to find solutions, either in eight-dimensional supergravity or in M-theory, with an $F_{4}$ flux on the supersymmetric four-cycle. This solution would make complete sense quantum mechanically, appart from being dual to a theory with Chern-Simons term. Another direction one would like to explore is the more interesting case of four-dimensional gauge theory arising from M-theory compactifications on $G_{2}$ holonomy manifolds. In this case one would like to understand the dynamical singularity resolution mechanism analog to the one discussed in sections 4 and 5 .

\section{Note added:}

While this paper was in preparation we received [104] which overlaps with some results of section 4 . However their results have been obtained using a different approach.

\section{Acknowledgements}

We would like to thank Ofer Aharony, Pascal Bain, Dan Freedman, Jerome Gauntlett, Gary Gibbons, Jaume Gomis, Joaquim Gomis, Amihay Hanany, Dario Martelli, Ventaka 
Nemani, Adam Ritz, James Sparks, David Tong and Paul Townsend for helpful discussions. We would like to thank Angel Paredes and Alfonso Ramallo for valuable comments on the manuscript. The work of U.G. and M.S. is supported in part by funds provided by the U.S. Department of Energy (D.O.E.) under cooperative research agreement \#DF-FC0294ER40818. M.S. is also supported in part by Fundación Antorchas and The British Council. The work of C.N. is supported by PPARC. 


\section{Appendix A: $D=8$ Salam-Sezgin's gauged supergravity}

The bosonic part of the $D=11$ supergravity Lagrangian [105 in tangent space is given by

$$
L_{D=11}=\frac{V}{4 \kappa^{2}} R_{(11)}-\frac{V}{48} F_{A B C D} F^{A B C D}+\frac{2 \kappa}{144^{2}} \epsilon^{A_{1}, \cdots A_{11}} F_{A_{1}, \ldots} F_{\ldots} V_{\cdots A_{11}}
$$

We will borrow the notation of [35] where $V \equiv \operatorname{det} V_{M}^{A}$ and the four-form field strength $F_{A B C D}=4 \partial_{[A} V_{B C D]}+12 \omega_{[A B}^{E} V_{C D] E}$. Indices $A, B=0, \cdots, 10$ are flat, while $M, N=0, \cdots, 10$ are curved. $V_{C D E}$ is the $D=11$ torsion-free spin connection. The metric signature is taken to be mostly plus and we will set $\kappa=1$ in what follows.

By dimensional reduction on $S^{3}, L_{D=11}$ becomes the $D=8$ Lagrangian obtained by Salam and Sezgin [35]. The total field content of the theory is

$$
\left(g_{\mu \nu}, \psi_{\mu}^{E}, B_{\mu \nu \rho}, B_{\mu \nu \alpha}, A_{\mu}^{\alpha}, B_{\mu \alpha}, \chi_{i}^{E}, L_{\alpha}^{i}, B, \phi\right)
$$

where $E=1,2$ and $\alpha, \beta, \ldots,=8,9,10$ and $i, j, \ldots=8,9,10$ are respectively curved and flat indices running over $S^{3}$, whereas $\mu, \nu, \ldots,=0, \ldots, 7$ and $a, b, \ldots=0, \ldots, 7$ are curved and flat respectively. $L_{\alpha}^{i}$ is a representative of the coset space $S L(3, R) / S O(3)$. Index $\alpha$ labels the global $S L(3, R)$ and the index $i$ labels the composite $S O(3)$ [35]. Together with $B$ and $\phi$ these constitute 7 scalars of the theory. One can further obtain a stable reduction down to the bosonic content,

$$
\left(g_{\mu \nu}, B_{\mu \nu \rho}, A_{\mu}^{\alpha}, L_{\alpha}^{i}, \phi\right)
$$

which is the background that we consider in our solutions. The bosonic part of $L_{D=8}$ for this background is,

$$
\begin{aligned}
e^{-1} L_{D=8}= & \frac{1}{4} R_{(8)}-\frac{1}{4} e^{2 \phi} F_{\mu \nu}^{\alpha} F^{\mu \nu \beta} g_{\alpha \beta}-\frac{1}{4} P_{\mu i j} P^{\mu i j}-\frac{1}{2} \partial_{\mu} \phi \partial^{\mu} \phi \\
& -\frac{1}{16} g_{c}^{2} e^{-2 \phi}\left(T_{i j} T^{i j}-\frac{1}{2} T^{2}\right)-\frac{1}{48} e^{2 \phi} G_{\mu \nu \rho \sigma} G^{\mu \nu \rho \sigma} .
\end{aligned}
$$

Here there is a word of notation. We define $e \equiv \operatorname{det} e_{\mu}^{\alpha}, e_{\mu}^{\alpha}$ being the vielbein and $R_{(8)}$ is the curvature scalar in $D=8$.

The $S U(2)$ two-form field strength is $F_{\mu \nu}^{\alpha}=\partial_{\mu} A_{\nu}^{\alpha}-\partial_{\nu} A_{\mu}^{\alpha}+g_{c} \epsilon^{\alpha \beta \gamma} A_{\mu}^{\beta} A_{\nu}^{\gamma}$ and the kinetic term for $L_{\alpha}^{i}$ is the symmetric traceless part of

$$
P_{\mu i j}+Q_{\mu i j}=L_{i}^{\alpha}\left(\partial_{\mu} \delta_{\alpha}^{\beta}-g_{c} \epsilon^{\alpha \beta \gamma} A_{\mu}^{\gamma}\right) L_{\beta j}
$$

while $Q_{\mu i j}$ denotes the anti-symmetric part.

We should mention that the dimensional reduction of the curvature scalar implies the dimensional reduction of the spin connection. Since the latter is defined in terms of the 
vielbein and its inverse, it is convenient to consider the reduction ansatz for the $D=11$ vielbein to the $D=8$

$$
V_{M}^{A}=\left(\begin{array}{cc}
e^{-\phi / 3} e_{\mu}^{a} & 0 \\
2 e^{2 \phi / 3} A_{\mu}^{\alpha} L_{\alpha}^{i} & e^{2 \phi / 3} L_{\alpha}^{i}
\end{array}\right) .
$$

Using this gauge breaks the $S O(1,10)$ Lorentz group down to $S O(1,7) \times S O(3)$. Other quantities which appear in the scalar potential are $T^{i j} \equiv L_{\alpha}^{i} L_{\beta}^{j} \delta^{\alpha \beta}$ and $T \equiv \delta_{i j} T^{i j}$. Finally, we define the four-form field strength as $G_{\mu \nu \rho \sigma}=\left(\partial_{\mu} B_{\nu \rho \sigma}+3\right.$ permutations $)+\left(2 F_{\mu \nu}^{\alpha} B_{\rho \sigma \alpha}+\right.$ 5 permutations).

The equations of motion for the system read,

$$
\begin{aligned}
R_{\mu \nu}= & P_{\mu}^{i j} P_{\nu}^{i j}+2 \partial_{\mu} \phi \partial_{\nu} \phi+2 e^{2 \phi} F_{\mu \lambda}^{i} F_{\nu}^{\lambda, i}-\frac{1}{3} g_{\mu \nu} \square \phi \\
& +\frac{1}{3} e^{2 \phi}\left(G_{\mu \lambda \tau \sigma} G_{\nu}^{\lambda \tau \sigma}-\frac{1}{12} g_{\mu \nu} G_{\rho \lambda \tau \sigma} G^{\rho \lambda \tau \sigma}\right), \\
\partial_{\mu}\left(\sqrt{-g} P^{\mu i j}\right)= & -\frac{2}{3} \square \phi \delta^{i j}+e^{2 \phi} F_{\mu \nu}^{i} F^{\mu \nu, j}+\frac{1}{36} e^{2 \phi} G_{\mu \nu \rho \lambda} G^{\mu \nu \rho \lambda} \delta^{i j} \\
& +\frac{1}{2} g_{c}^{2} e^{-2 \phi}\left[T_{n}^{i} T_{n}^{j}-\frac{1}{2} T T^{i j}-\frac{1}{2} \delta^{i j}\left(T_{m n} T^{m n}-\frac{1}{2} T^{2}\right)\right], \\
\partial_{\mu}\left(\sqrt{-g} e^{2 \phi} F^{\mu \nu, i}\right)= & -e^{2 \phi} P_{\mu}^{i j} F^{\mu \nu, j}-g_{c} g^{\mu \nu} \epsilon^{i j k} P_{\mu}^{j l} T_{k l}, \\
\partial_{\mu}\left(\sqrt{-g} e^{2 \phi} G^{\mu \nu \rho \sigma}\right)= & 0 .
\end{aligned}
$$

As shown in 35], above equations of motion in $D=8$ can be uplifted to $D=11 \mathrm{EOM}$ derived from the Cremmer-Julia-Scherk supergravity [105]. In particular, compactifications of $D=11$ supergravity containing either a three-dimensional manifold as a part of the seven-dimensional product space, or as a fiber space in a seven-dimensional fiber bundle, are also compactified solutions of $D=8$ supergravity. One necessary condition in order to have these kind of solutions is that $S U(2)$ coupling constant $g_{c}$ is non-vanishing. To get supersymmetric solutions, the gauge connection of the normal bundle on a four-cycle $B^{4}$ (in the seven-dimensional $B^{4} \times S^{3}$ space-time) must be equal to the spin connection of the spin bundle of $B^{4}$.

\section{Appendix B: General Solutions}

In this appendix we report about more general analytic solutions of BPS which contain (17) and (39) as special solutions. Some of these solutions are physical by Gubser's criterion as we will demonstrate below, hence they will correspond to RG flows in the dual gauge theory. However, field theory interpretation is not clear to us at the moment of writing this manuscript.

For the case of the $\mathcal{N}=1$ system, as a first step to obtain the general solution, let us define the field $x \equiv 20 e^{2 \phi-2 h}$. The differential equation for $x$ in the variable $d / d t=e^{\phi} d / d r$, 
is solved as,

$$
x(t)=\frac{1}{1+a e^{-t / 2}} .
$$

where $a$ is an integration constant. Note that $a=0$ corresponds to the special solution (17). $a<0$ case will be unacceptable by Gubser's criterion, hence we consider $a>0$ for which, from (12)-(14), we can find the general solution,

$$
\begin{aligned}
& h(t)=\frac{1}{4} \log \left(\frac{4 \Lambda}{5}(c-I(v))\right)+\frac{t}{8}+\frac{1}{5} \log \left(a+e^{t / 2}\right)-\frac{9}{20} \log (a), \\
& \phi(t)=h(t)-\frac{1}{2} \log \left(20\left(1+a e^{-t / 2}\right)\right) .
\end{aligned}
$$

where $I(t)$ is defined as,

$$
\begin{aligned}
I(v)= & \left\{\frac{5 v}{1-v^{5}}-4 \log (1-v)\right. \\
& +2 \sqrt{2(5+\sqrt{5})} \arctan \left(\frac{4 v-(\sqrt{5}-1)}{\sqrt{2(5+\sqrt{5})}}\right) \\
& +2 \sqrt{2(5-\sqrt{5})} \arctan \left(\frac{4 v+(\sqrt{5}+1)}{\sqrt{2(5-\sqrt{5})}}\right) \\
& -(\sqrt{5}-1) \log \left(v^{2}-\frac{1}{2}(\sqrt{5}-1) v+1\right) \\
& \left.+(\sqrt{5}+1) \log \left(v^{2}+\frac{1}{2}(\sqrt{5}+1) v+1\right)\right\},
\end{aligned}
$$

where $v=\left(\frac{e^{t / 2}}{a}+1\right)^{1 / 5}$.

In order to obtain the fixed point solution (22) in the limit $t \rightarrow \infty$, the integration constant of $h(t)$ should be chosen as $c=\pi(\sqrt{2(5-\sqrt{5})}+\sqrt{2(5+\sqrt{5})})$.

On the other extremun of the flow, $t \rightarrow-\infty$ we can read the asymptotic expansions of the fields and the eleven-dimensional solutions to be

$$
h \approx \frac{1}{4} \log (4 \Lambda), \quad \phi \approx t / 4+\frac{1}{4} \log (\Lambda / 100), \quad f \approx t / 4,
$$

and in the variable $d \rho=\frac{\Lambda}{100} e^{t / 6} d t$ such that $t \rightarrow-\infty$ is $\rho=0$

$$
d s_{11}^{2}=\rho^{2} d x_{1,2}^{2}+\frac{6}{\rho}\left(\frac{80^{2} \Lambda^{3}}{100}\right)^{1 / 6} d \Omega_{4}^{2}+\frac{\rho^{2}}{36}\left(\omega^{i}-A^{i}\right)^{2}+d \rho^{2},
$$

and, in flat indexes,

$$
F_{x y t \rho} \approx 1 / \rho
$$


A fact that we would like to remark is that, if we do not impose the integration constant to be $c=\pi(\sqrt{2(5-\sqrt{5})}+\sqrt{2(5+\sqrt{5})})$ the solution "misses" the fixed point and for large values of the radial variable $t$ it asymptotically approaches a cone over weak $G_{2}$ manifold, having an expression as Eq.(20).

The solution above has a curvature singularity for small values of the radial coordinate $\rho$. We should be able to decide whether we should accept or not the singular behaviour. In order to do that, we can "integrate out" the degrees of freedom living on the four-sphere and the gauge fields in the eight-dimensional supergravity Lagrangian and transform the system into one of gravity plus scalars.

Having done this, we find a four-dimensional Lagrangian that reads

$$
L=\sqrt{g_{4}}\left[R+T-V_{e f f}\right],
$$

where, $T$ denotes kinetic terms for the fields $\phi, h$ and $V_{\text {eff }}$ is given by

$$
V_{e f f}=-3 e^{-6 h}-\frac{\Lambda^{2}}{2} e^{-12 h-2 \phi}-\frac{3}{32} e^{-2 \phi-4 h}+\frac{9}{4} e^{2 \phi-8 h} .
$$

We can see that near this singularity, this potential is bounded above, this renders the singularity to be acceptable according to the criterion introduced in 85. We conclude that UV of the dual field theory is at $t=+\infty$ for which the geometry is $A d S_{4} \times \tilde{S}_{7}$. IR is at $t=-\infty$ for which one obtains above asymptotic geometry. Note that in $\rho \rightarrow 0$ limit of (107), $S^{4}$ radius becomes much larger than size of the domain-wall. This fact renders field theory interpretation difficult in eleven dimensions. However, considering the eightdimensional supergravity problem, one does not run into trouble with the nature of the singularity at $t \rightarrow-\infty$ which is acceptable by above criteria and corresponds to the IR limit of the corresponding field theory. Therefore, one can conclude that $t \rightarrow \infty \operatorname{limit}\left(\operatorname{Ad} S_{4} \times S_{4}\right)$ corresponds to UV and $t \rightarrow-\infty$ limit of eight-dimensional super-kink corresponds to IR of the dual $\mathcal{N}=1 \mathrm{SYM}$ in three dimensions.

For the case of $\mathcal{N}=2$, similarly we first introduce the field, $x \equiv 4 e^{2 \phi-2 h+2 \lambda}$ which can be solved in the variable $d / d t=e^{\phi+4 \lambda} d / d r$ as in (102). A more general solution than (39) can be obtained however by keeping $x=1$ frozen, but turning on $\lambda$ field:

$$
\begin{aligned}
\lambda(t) & =\frac{1}{6} \log \left(\frac{2 e^{2 t}}{e^{2 t}-1}\right) \\
e^{4 h(t)-4 \lambda(t)} & =\Lambda\left(\log \left(\frac{e^{t / 2}+1}{e^{t / 2}-1}\right)+2 \arctan \left(e^{t / 2}\right)-C\right) e^{-t / 2}\left(e^{2 t}-1\right) \\
\phi(t) & =h(t)-\lambda(t)-\log (2) \\
f(t) & =-\frac{t}{24}+\frac{1}{12} \log \left(e^{2 t}-1\right)-\frac{1}{4} \log \left(\log \left(\frac{e^{t / 2}+1}{e^{t / 2}-1}\right)+2 \arctan \left(e^{t / 2}\right)-C\right)
\end{aligned}
$$


where for convinience we fixed the integration constant for $\lambda$. If constant $C$ above is chosen as $C=\pi$, then the solution reaches the fixed point solution of Eq.(46).

On the other hand, if we do not impose this value for the integration constant, again as in the $\mathcal{N}=1$ case, the solution misses the fixed point. Then, for large values of the radial variable $t$ the functions $f, h, \lambda, \phi$ approach

$$
\lambda=1 / 6 \log (2), \quad \phi \approx h \approx 3 f \approx 3 / 8 t
$$

Thus leading to a metric of the form $E^{2,1} \times C Y 4$ like in Eq.(37).

On the other extremun of the flow $(t \rightarrow 0)$, there is a curvature singularity as in $\mathcal{N}=1$

case. We can also define a similar effective Lagrangian, but in this case the kinetic terms will include the field $\lambda$. The effective potential reads

$$
V_{e f f}=-e^{-6 h}-\frac{\Lambda^{2}}{2} e^{-2 \phi-12 h}+e^{2 \phi-8 h-4 \lambda}+\frac{g^{2}}{32} e^{-2 \phi-4 h}\left(e^{-8 \lambda}-2 e^{-2 \lambda}\right) .
$$

If we analyze the behaviour of this effective potential near the IR, we see that it is again bounded above, rendering the singularity acceptable. One can conclude that $t \rightarrow \infty$ limit $\left(A d S_{4} \times S_{2} \times S_{2}\right)$ corresponds to UV and $t \rightarrow 0$ limit of eight-dimensional super-kink corresponds to IR of the dual $\mathcal{N}=2 \mathrm{SYM}$ in three dimensions.

Yet a most general solution can be found analytically by turning on $x$ defined above (solution will be Eq.(102)). It is also acceptable by means Gubser's criterion for some ranges of the integration constants.

\section{Appendix C: Orbits of BPS equations: $\mathcal{N}=1$ case}

Although we were able to find analytical solutions of the BPS equations for the situations studied in this paper, it is interesting to see how this solutions behave as orbits of the BPS equations. Here we show the orbits for the case preserving two supercharges, i.e. our first example. The other cases for $\mathcal{N}=2$ and $\mathcal{N}=3$ are similar since for such cases we have considered situations with only irrelevant deformations, such that for instance $\lambda$ is fixed so that at the end we can reduce the analysis to one orbit equation as Eq.(27).

For the present case we consider the orbits of Eq.(27). We recall that we are doing a similar analysis as in [59]. In figure 6 we plot some numerical solutions of Eq.(27). The straight line $H=20 \mathrm{~s}$ represents a collection of IR fixed points if we consider flowing down from a Spin(7) manifold at UV, for different values of $\Lambda$. As an example we plot a short solid line segment indicating this kind of flow that is the same as described in figure 2 in the body of the paper. The rest of solid lines correspond to solutions for non-vanishing $\Lambda$, which are "missing" IR fixed points. At UV all of these curves approach $H=20 \mathrm{~s}$ leading to the metric of $E^{2,1} \times \operatorname{Spin}(7)$. All them approach the origin with the same slope. They correspond to 
some of the solutions included in Appendix B. Indeed, the almost vertical solid line around the point labeled as IR f.p. is one of the solutions introduced in Appendix B for which we do not have a clear physical interpretation at the present. Particularly, for this solution the IR/UV limits are the opposite as labeled in figure 6.

The dashed line represents a pure-metric solution, i.e. when $\Lambda=0$.

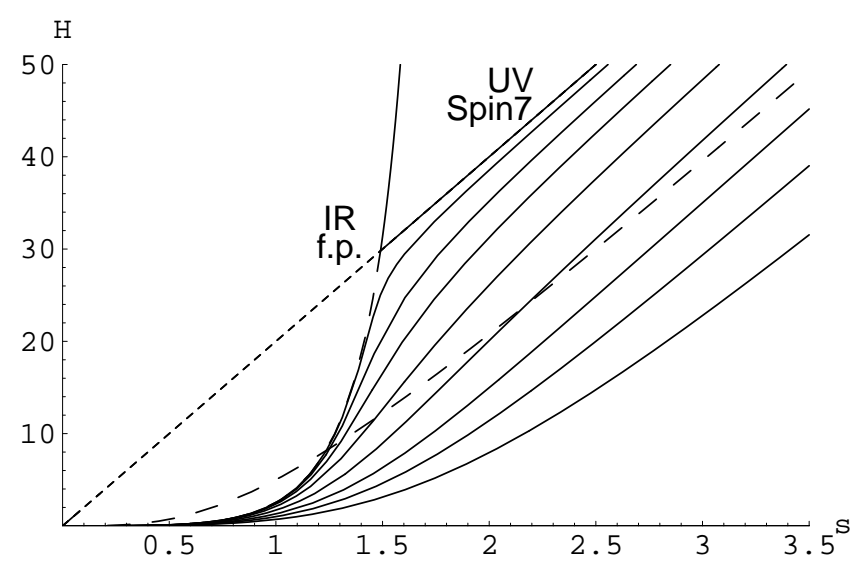

Figure 6: Orbits for BPS equations for $\mathcal{N}=1$ case.

\section{Appendix D: Notation for the left-invariant one-forms}

In the definition of the squashed seven-sphere we have used the left-invariant one-forms as follows. For the three-sphere in $S^{4}$ we used

$$
\begin{aligned}
\sigma^{1} & =\cos \psi d \theta+\sin \psi \sin \theta d \phi \\
\sigma^{2} & =\sin \psi d \theta-\cos \psi \sin \theta d \phi \\
\sigma^{3} & =d \psi+\cos \theta d \phi
\end{aligned}
$$

while for the $S U(2)$ group manifold we have used

$$
\begin{aligned}
\omega^{1} & =\cos \gamma d \alpha+\sin \gamma \sin \alpha d \beta, \\
\omega^{2} & =\sin \gamma d \alpha-\cos \gamma \sin \alpha d \beta \\
\omega^{3} & =d \gamma+\cos \alpha d \beta .
\end{aligned}
$$




\section{References}

[1] M. Atiyah, J. Maldacena and C. Vafa, "An M-theory flop as a large N duality," J. Math. Phys. 42, 3209 (2001) [arXiv:hep-th/0011256].

[2] M. Atiyah and E. Witten, "M-theory dynamics on a manifold of G(2) holonomy," arXiv:hep-th/0107177.

[3] S. Gukov and J. Sparks, "M-theory on Spin(7) manifolds. I," arXiv:hep-th/0109025.

[4] B. S. Acharya, "Confining strings from G(2)-holonomy spacetimes," arXiv:hepth/0101206.

[5] M. Cvetic, G. W. Gibbons, H. Lu and C. N. Pope, "Hyper-Kaehler Calabi metrics, $\mathrm{L}^{* *} 2$ harmonic forms, resolved M2-branes, and $\operatorname{AdS}(4) / \mathrm{CFT}(3)$ correspondence," Nucl. Phys. B 617, 151 (2001) [arXiv:hep-th/0102185].

[6] B. S. Acharya and C. Vafa, "On domain walls of $\mathrm{N}=1$ supersymmetric Yang-Mills in four dimensions," arXiv:hep-th/0103011.

[7] J. Gomis, "D-branes, holonomy and M-theory," Nucl. Phys. B 606, 3 (2001) [arXiv:hepth/0103115].

[8] M. Cvetic, G. W. Gibbons, H. Lu and C. N. Pope, "New complete non-compact Spin(7) manifolds," Nucl. Phys. B 620, 29 (2002) [arXiv:hep-th/0103155].

[9] M. Cvetic, G. W. Gibbons, H. Lu and C. N. Pope, New Cohomogeneity One Metrics With $\operatorname{Spin}(7)$ Holonomy math.DG/0105119

[10] J. D. Edelstein and C. Nunez, "D6 branes and M-theory geometrical transitions from gauged supergravity," JHEP 0104, 028 (2001) [arXiv:hep-th/0103167].

[11] A. Brandhuber, J. Gomis, S. S. Gubser and S. Gukov, "Gauge theory at large N and new G(2) holonomy metrics," Nucl. Phys. B 611, 179 (2001) [arXiv:hep-th/0106034].

[12] M. Cvetic, G. W. Gibbons, H. Lu and C. N. Pope, "Resolved branes and M-theory on special holonomy spaces," arXiv:hep-th/0106177.

[13] J. Gomis and T. Mateos, "D6 branes wrapping Kaehler four-cycles," Phys. Lett. B 524, 170 (2002) [arXiv:hep-th/0108080].

[14] M. Cvetic, G. W. Gibbons, H. Lu and C. N. Pope, "Ricci-flat metrics, harmonic forms and brane resolutions," arXiv:hep-th/0012011.

[15] E. Witten, "Anomaly cancellation on G(2)manifolds," arXiv:hep-th/0108165. 
[16] M. Cvetic, G. W. Gibbons, H. Lu and C. N. Pope, "Cohomogeneity one manifolds of Spin(7) and G(2) holonomy," arXiv:hep-th/0108245.

[17] B. Acharya and E. Witten, "Chiral fermions from manifolds of $G(2)$ holonomy," arXiv:hep-th/0109152.

[18] R. Blumenhagen and V. Braun, "Superconformal field theories for compact G(2) manifolds," JHEP 0112, 006 (2001) [arXiv:hep-th/0110232]; R. Blumenhagen and V. Braun, "Superconformal field theories for compact manifolds with Spin(7) holonomy," JHEP 0112, 013 (2001) [arXiv:hep-th/0111048].

[19] J. Gomis, "On SUSY breaking and chiSB from string duals," Nucl. Phys. B 624, 181 (2002) [arXiv:hep-th/0111060].

[20] G. Curio, B. Kors and D. Lust, "Fluxes and branes in type II vacua and M-theory geometry with G(2) and Spin(7) holonomy," arXiv:hep-th/0111165.

[21] M. Cvetic, G. Shiu and A. M. Uranga, "Chiral type II orientifold constructions as M theory on G(2) holonomy spaces," arXiv:hep-th/0111179.

[22] M. Cvetic, G. W. Gibbons, H. Lu and C. N. Pope, "M-theory conifolds," arXiv:hepth/0112098.

[23] A. Brandhuber, "G(2) holonomy spaces from invariant three-forms," arXiv:hepth/0112113.

[24] K. Sfetsos, "Integrable reductions of $\operatorname{Spin}(7)$ and G(2) invariant self-dual Yang-Mills equations and gravity," arXiv:hep-th/0112117.

[25] M. Cvetic, G. W. Gibbons, H. Lu and C. N. Pope, "A G(2) unification of the deformed and resolved conifolds," arXiv:hep-th/0112138.

[26] R. Hernandez and K. Sfetsos, "An eight-dimensional approach to G(2) manifolds," arXiv:hep-th/0202135.

[27] S. Gukov and D. Tong, "D-brane probes of G(2) holonomy manifolds," arXiv:hepth/0202125.

[28] S. Gukov and D. Tong, "D-brane probes of special holonomy manifolds, and dynamics of $\mathrm{N}=1$ three-dimensional gauge theories," arXiv:hep-th/0202126.

[29] G. T. Horowitz and A. Strominger, "Black Strings And P-Branes," Nucl. Phys. B 360, 197 (1991). 
[30] T. Eguchi and A. J. Hanson, "Asymptotically Flat Selfdual Solutions To Euclidean Gravity," Phys. Lett. B 74, 249 (1978).

[31] R. Bryant and S. Salamon, "On the construction of some Complete Metrics with Exceptional Holonomy," Duke Math. J. 58 (1989) 829. G. W. Gibbons, D. N. Page and C. N. Pope, "Einstein Metrics On $\mathrm{S}^{* *} 3 \mathrm{R}^{* *} 3$ And $\mathrm{R}^{* *} 4$ Bundles," Commun. Math. Phys. 127, 529 (1990).

[32] E. Calabi, "Métriques kählériennes et fibrés holomorphes," Ann. Scient. Ecole Norm. Sup. 12, (1979) 269.

[33] R. Hernandez, "Branes wrapped on coassociative cycles," Phys. Lett. B 521, 371 (2001) [arXiv:hep-th/0106055].

[34] S. S. Gubser, "TASI lectures: Special holonomy in string theory and M-theory," arXiv:hep-th/0201114.

[35] A. Salam and E. Sezgin, "D = 8 Supergravity," Nucl. Phys. B 258, 284 (1985).

[36] B. Acharya, X. de la Ossa and S. Gukov, "G-flux, Supersymmetry and Spin(7) manifolds," arXiv:hep-th/0201227.

[37] D. Berenstein, J. Maldacena and H. Nastase, "Strings in flat space and pp waves from $\mathrm{N}=4$ super Yang Mills," arXiv:hep-th/0202021.

[38] R. R. Metsaev and A. A. Tseytlin, "Exactly solvable model of superstring in plane wave Ramond-Ramond background," arXiv:hep-th/0202109.

[39] M. Blau, J. Figueroa-O'Farrill and G. Papadopoulos, "Penrose limits, supergravity and brane dynamics," arXiv:hep-th/0202111.

[40] D. Berenstein, C. P. Herzog and I. R. Klebanov, "Baryon spectra and AdS/CFT correspondence," arXiv:hep-th/0202150.

[41] N. Itzhaki, I. R. Klebanov and S. Mukhi, "PP wave limit and enhanced supersymmetry in gauge theories," arXiv:hep-th/0202153.

[42] J. Gomis and H. Ooguri, "Penrose limit of N = 1 gauge theories," arXiv:hep-th/0202157.

[43] J. G. Russo and A. A. Tseytlin, "On solvable models of type IIB superstring in NS-NS and R-R plane wave backgrounds," arXiv:hep-th/0202179.

[44] L. A. Zayas and J. Sonnenschein, "On Penrose limits and gauge theories," arXiv:hepth/0202186. 
[45] M. Hatsuda, K. Kamimura and M. Sakaguchi, "From super-AdS(5) x S**5 algebra to super-pp-wave algebra," arXiv:hep-th/0202190.

[46] M. Alishahiha and M. M. Sheikh-Jabbari, "The PP-wave limits of orbifolded AdS(5) x S**5," arXiv:hep-th/0203018.

[47] M. Billo' and I. Pesando, "Boundary states for GS superstrings in an Hpp wave background," arXiv:hep-th/0203028.

[48] N. w. Kim, A. Pankiewicz, S. J. Rey and S. Theisen, "Superstring on pp-wave orbifold from large-N quiver gauge theory," arXiv:hep-th/0203080.

[49] M. Cvetic, H. Lu and C. N. Pope, "Penrose Limits, PP-Waves and Deformed M2branes," arXiv:hep-th/0203082.

[50] T. Takayanagi and S. Terashima, "Strings on orbifolded pp-waves," arXiv:hepth/0203093.

[51] E. Floratos and A. Kehagias, "Penrose limits of orbifolds and orientifolds," arXiv:hepth/0203134.

[52] M. Cvetic, H. Lu and C. N. Pope, "Gauged six-dimensional supergravity from massive type IIA," Phys. Rev. Lett. 83, 5226 (1999) [arXiv:hep-th/9906221].

[53] O. Pelc and R. Siebelink, "The D2-D6 system and a fibered AdS geometry," Nucl. Phys. B 558, 127 (1999) [arXiv:hep-th/9902045].

[54] K. A. Intriligator and N. Seiberg, "Mirror symmetry in three dimensional gauge theories," Phys. Lett. B 387, 513 (1996) [arXiv:hep-th/9607207].

[55] J. de Boer, K. Hori, H. Ooguri and Y. Oz, "Mirror symmetry in three-dimensional gauge theories, quivers and D-branes," Nucl. Phys. B 493, 101 (1997) [arXiv:hep-th/9611063].

[56] M. Bershadsky, C. Vafa and V. Sadov, "D-Branes and Topological Field Theories," Nucl. Phys. B 463, 420 (1996) [arXiv:hep-th/9511222].

[57] J. Maldacena and C. Núñez, "Supergravity description of field theories on curved manifolds and a no go theorem," Int. J. Mod. Phys. A 16, 822 (2001) [arXiv:hep-th/0007018].

[58] J. M. Maldacena and C. Núnez, "Towards the large $N$ limit of pure $\mathcal{N}=1$ super Yang-Mills," Phys. Rev. Lett. 86 (2001) 588 [arXiv:hep-th/0008001].

[59] B. S. Acharya, J. P. Gauntlett and N. Kim, "Fivebranes wrapped on associative threecycles," Phys. Rev. D 63, 106003 (2001) [arXiv:hep-th/0011190]. 
[60] H. Nieder and Y. Oz, "Supergravity and D-branes wrapping special Lagrangian cycles," JHEP 0103, 008 (2001) [arXiv:hep-th/0011288].

[61] J. P. Gauntlett, N. Kim and D. Waldram, "M-fivebranes wrapped on supersymmetric cycles," Phys. Rev. D 63, 126001 (2001) [arXiv:hep-th/0012195].

[62] C. Núñez, I. Y. Park, M. Schvellinger and T. A. Tran, "Supergravity duals of gauge theories from $F(4)$ gauged supergravity in six dimensions," JHEP 0104, 025 (2001) [arXiv:hep-th/0103080].

[63] M. Schvellinger and T. A. Tran, "Supergravity duals of gauge field theories from SU(2) x U(1) gauged supergravity in five dimensions," JHEP 0106, 025 (2001) [arXiv:hepth/0105019].

[64] J. P. Gauntlett, N. Kim, S. Pakis and D. Waldram, "Membranes wrapped on holomorphic curves," Phys. Rev. D 65, 026003 (2002) [arXiv:hep-th/0105250].

[65] Y. Imamura, "1/4 BPS solutions in massive IIA supergravity," Prog. Theor. Phys. 106, 653 (2001) [arXiv:hep-th/0105263].

[66] J. P. Gauntlett, N. Kim, D. Martelli and D. Waldram, "Wrapped fivebranes and N = 2 super Yang-Mills theory," Phys. Rev. D 64, 106008 (2001) [arXiv:hep-th/0106117].

[67] J. P. Gauntlett and N. Kim, "M-fivebranes wrapped on supersymmetric cycles. II," arXiv:hep-th/0109039.

[68] T. A. Tran, "Gauged supergravities from spherical reductions," arXiv:hep-th/0109092.

[69] J. Gomis and J. G. Russo, "D $=2+1 \mathrm{~N}=2$ Yang-Mills theory from wrapped branes," JHEP 0110, 028 (2001) [arXiv:hep-th/0109177].

[70] J. P. Gauntlett, N. w. Kim, D. Martelli and D. Waldram, "Fivebranes wrapped on SLAG three-cycles and related geometry," JHEP 0111, 018 (2001) [arXiv:hep-th/0110034].

[71] P. Di Vecchia, H. Enger, E. Imeroni and E. Lozano-Tellechea, "Gauge theories from wrapped and fractional branes," arXiv:hep-th/0112126.

[72] J. P. Gauntlett, N. Kim, S. Pakis and D. Waldram, "M-Theory solutions with AdS factors," arXiv:hep-th/0202184.

[73] H. J. Boonstra, K. Skenderis and P. K. Townsend, "The domain wall/QFT correspondence," JHEP 9901, 003 (1999) [arXiv:hep-th/9807137].

[74] L. Jarv and C. V. Johnson, "Orientifolds, M-theory, and the ABCD's of the enhancon," Phys. Rev. D 62, 126010 (2000) [arXiv:hep-th/0002244]. 
[75] D. Joyce, Compact Manifolds With Special Holonomy, (Oxford University Press, 2000).

[76] M. B. Green, J. A. Harvey and G. W. Moore, "I-brane inflow and anomalous couplings on D-branes," Class. Quant. Grav. 14, 47 (1997) [arXiv:hep-th/9605033].

[77] M.J. Duff, H. Lü, C.N. Pope, E. Sezgin, "Supermembranes with fewer supersymmetries," Phys. Lett. B 371, 206 (1996) [arXiv:hep-th/9511162].

[78] L. Castellani, A. Ceresole, D. D'Auria, S. Ferrara, P. Fre', "G/H M-Branes and AdS(p+2) geometries," Nucl. Phys. B 527, 442 (1998) [arXiv:hep-th/9803039].

[79] M.J. Duff, B.E.W. Nilsson, C.N. Pope, "Squashed supersymmetry breaking by the squashed seven sphere," Phys. Rev. Lett. 50, 2043 (1983)

[80] D. S. Freed and E. Witten, "Anomalies in string theory with D-branes," arXiv:hepth/9907189.

[81] E. Witten, "On flux quantization in M-theory and the effective action," J. Geom. Phys. 22, 1 (1997) [arXiv:hep-th/9609122].

[82] I.R. Klebanov and E. Witten, "ADS/CFT correspondence and symmetry breaking," Nucl. Phys. B 556, 89 (1999) [arXiv:hep-th/9905104].

[83] C. Ahn and S. Rey, "Three dimensional CFT's and RG flow from squashing M2-brane horizon," Nucl. Phys. B 565, 210 (2000) [arXiv:hep-th/9908110].

[84] M.J. Duff, B.E.W. Nilsson, C.N. Pope, "Kaluza-Klein supergravity," Phys. Rept. 130, $1(1986)$

[85] S. S. Gubser, "Curvature singularities: The good, the bad, and the naked," arXiv:hepth/0002160.

[86] P. Merlatti, "M-theory on $\operatorname{AdS}(4)$ x Q(111): The complete $\operatorname{Osp}(2 \mid 4)$ x SU(2) x SU(2) x SU(2) spectrum from harmonic analysis," Class. Quant. Grav. 18, 2797 (2001) [arXiv:hep-th/0012159].

[87] D. Fabbri, P. Fre', L. Gualtieri, C. Reina, A. Tomasiello, A. Zaffaroni and A. Zampa, "3-D superconformal field theories from Sasakian seven manifolds: New nontrivial evidences for AdS(4)/CFT(3)," Nucl. Phys. B 577, 547 (2000) [arXiv:hep-th/9907219];

[88] D. Fabbri, "Three dimensional conformal field theories from Sasakian seven-manifolds," arXiv:hep-th/0002255.

[89] G. Dall'Agata, "N = 2 conformal field theories from M2-branes at conifold singularities," Phys. Lett. B 460, 79 (1999) [arXiv:hep-th/9904198]. 
[90] C. h. Ahn, "N = 2 SCFT and M theory on $\operatorname{AdS}(4)$ x $Q(1,1,1)$," Phys. Lett. B 466, 171 (1999) [arXiv:hep-th/9908162].

[91] K. Oh and R. Tatar, "Three dimensional SCFT from M2 branes at conifold singularities," JHEP 9902, 025 (1999) [arXiv:hep-th/9810244].

[92] D. Fabbri, P. Fre, L. Gualtieri and P. Termonia, "Osp(N|4) supermultiplets as conformal superfields on $\mathrm{d}(\operatorname{AdS}(4))$ and the generic form of $\mathrm{N}=2, \mathrm{D}=3$ gauge theories," Class. Quant. Grav. 17, 55 (2000) [arXiv:hep-th/9905134].

[93] B. S. Acharya, J. M. Figueroa-O'Farrill, C. M. Hull and B. Spence, "Branes at conical singularities and holography," Adv. Theor. Math. Phys. 2, 1249 (1999) [arXiv:hepth/9808014].

[94] D. R. Morrison and M. R. Plesser, "Non-spherical horizons. I," Adv. Theor. Math. Phys. 3, 1 (1999) [arXiv:hep-th/9810201].

[95] C. P. Herzog and I. R. Klebanov, "Gravity duals of fractional branes in various dimensions," Phys. Rev. D 63, 126005 (2001) [arXiv:hep-th/0101020].

[96] A. Kapustin and M. J. Strassler, "On mirror symmetry in three dimensional Abelian gauge theories," JHEP 9904, 021 (1999) [arXiv:hep-th/9902033].

[97] P. Termonia, "The complete $\mathrm{N}=3$ Kaluza-Klein spectrum of 11D supergravity on $\operatorname{AdS}(4)$ x N(010)," Nucl. Phys. B 577, 341 (2000) [arXiv:hep-th/9909137].

[98] M. Billo, D. Fabbri, P. Fre, P. Merlatti and A. Zaffaroni, "Rings of short N = 3 superfields in three dimensions and M-theory on $\operatorname{AdS}(4)$ x N $(0,1,0)$," Class. Quant. Grav. 18, 1269 (2001) [arXiv:hep-th/0005219].

[99] L. J. Romans, "The F(4) Gauged Supergravity In Six-Dimensions," Nucl. Phys. B 269, 691 (1986).

[100] M. Petrini and A. Zaffaroni, "The holographic RG flow to conformal and non-conformal theory," arXiv:hep-th/0002172.

[101] V.L. Campos, G. Ferretti, H. Larsson, D. Martelli and B.E.W. Nilsson, "A study of holographic RG flows in $D=6$ and $D=3$," JHEP 0006, 023, (2000) [arXiv:hepth/0003151].

[102] D. Z. Freedman, S. S. Gubser, K. Pilch and N. P. Warner, "Renormalization group flows from holography supersymmetry and a c-theorem," Adv. Theor. Math. Phys. 3, 363 (1999) [arXiv:hep-th/9904017]. 
[103] D. Z. Freedman, S. S. Gubser, K. Pilch and N. P. Warner, "Continuous distributions of D3-branes and gauged supergravity," JHEP 0007, 038 (2000) [arXiv:hep-th/9906194].

[104] A. Loewy and Y. Oz, "Branes in Special Holonomy Backgrounds," arXiv:hepth/0203092.

[105] E. Cremmer, B. Julia and J. Scherk, "Supergravity Theory In 11 Dimensions," Phys. Lett. B 76, 409 (1978). 